\title{
Integral Formulas for Quantum Isomonodromic Systems
}

\author{
by
}

\author{
Hajime NAGOYA
}

\begin{abstract}
We present integral formulas for particular solutions of quantum isomonodromic systems, which are time-dependent Schrödinger systems and quantizations of isomonodromic deformations for certain Fuchsian systems. The functions given by these integral formulas are generalizations of the generalized hypergeometric function ${ }_{L} F_{L-1}$.
\end{abstract}

2010 Mathematics Subject Classification: Primary 33C70; Secondary $81 T 40$.

Keywords: quantization of isomonodromic systems, hypergeometric integrals, conformal field theory, quantum Painlevé systems, time-dependent Schrödinger systems.

\section{$\S 1$. Introduction}

Fix integers $L \geq 2$ and $N \geq 1$. We consider the time-dependent Schrödinger system

$$
\kappa \frac{\partial}{\partial z_{i}} \Psi(\mathbf{q}, \mathbf{z})=H_{i}\left(\mathbf{q}, \frac{\partial}{\partial \mathbf{q}}, \mathbf{z}\right) \Psi(\mathbf{q}, \mathbf{z}) \quad(1 \leq i \leq N)
$$

where $\kappa \in \mathbb{C}$ and $\Psi(\mathbf{q}, \mathbf{z})$ is an unknown function of

$$
\mathbf{q}=\left(q_{1}^{(1)}, \ldots, q_{L-1}^{(1)}, q_{1}^{(2)}, \ldots, q_{L-1}^{(2)}, \ldots, q_{1}^{(N)}, \ldots, q_{L-1}^{(N)}\right)
$$

and $\mathbf{z}=\left(z_{1}, \ldots, z_{N}\right)$. The Hamiltonians $H_{i}$ are defined in Definition 2.1.

The Schrödinger system (1.1) is a quantization of the classical Hamiltonian system $\mathcal{H}_{L, N}$ obtained from a similarity reduction of the Drinfeld-Sokolov hierarchy by K. Fuji and T. Suzuki [3] $(L=3, N=1)$, T. Suzuki [14] $(L \geq 2, N=1)$, and a similarity reduction of the UC hierarchy by $\mathrm{T}$. Tsuda [18] $(L \geq 2, N \geq 1)$, independently. In [18], T. Tsuda showed that the classical Hamiltonian system $\mathcal{H}_{L, N}$ is equivalent to a Schlesinger system governing isomonodromic deformations for a certain Fuchsian system.

Communicated by T. Mochizuki. Received September 23, 2012. Revised February 5, 2013.

H. Nagoya: Department of Mathematics, Rikkyo University, Toshima-ku, Tokyo 171-8501, Japan; e-mail: nagoya.hajime@rikkyo.ac.jp

(C) 2013 Research Institute for Mathematical Sciences, Kyoto University. All rights reserved. 
On the other hand, Y. Yamada conjectured in the context of the so-called AGT relation that the instanton partition function, in the presence of the full surface operator in $\mathcal{N}=2 S U(L)$ gauge theory, is determined by the Schrödinger system (1.1) for $N=1$ [19]. In the case of $L=2$, the Schrödinger system (1.1) is a quantization of the Garnier systems [4], [6], which has appeared in conformal field theory [16].

In this paper, we present a family of hypergeometric integrals as particular solutions to the Schr̈odinger system (1.1). These solutions are polynomials in $\mathbf{q}$ of degree $M \in \mathbb{Z}_{\geq 1}$ and their coefficients are integral representations of hypergeometric type.

A key to finding special solutions to quantum isomonodromic systems is to observe special solutions to the corresponding classical isomonodromic systems. For example, both the classical and quantum sixth Painlevé equation have a particular solution expressed in terms of the Gauss hypergeometric function [9].

It is known that the classical Hamiltonian system $\mathcal{H}_{L, N}$ has a particular solution expressed in terms of a generalization of the Gauss hypergeometric function by K. Okamoto and H. Kimura [11] $(L=2, N \geq 1)$, T. Suzuki [15] $(L \geq 2, N=1)$, T. Tsuda [17] ( $L \geq 2, N \geq 1)$. Observing the linear Pfaffian system derived from this generalization of the Gauss hypergeometric function, we see indeed that hypergeometric integrals given in [17] yield a particular solution to the Schrödinger system (1.1):

Theorem 1.1 (see Theorem 4.1). The hypergeometric integral

$$
\begin{aligned}
\int_{\Delta} \prod_{n=1}^{L-1} t_{n}^{\alpha_{n} / \kappa} \prod_{i=1}^{N}\left(1-z_{i} t_{L-1}\right)^{-\beta_{i} / \kappa} \prod_{n=1}^{L-1}\left(t_{n-1}-t_{n}\right)^{-\gamma_{n} / \kappa} \\
\\
\quad \times\left(\varphi_{0}(t)-\sum_{i=1}^{N} \sum_{n=1}^{L-1} \varphi_{n}^{(i)}(t) q_{n}^{(i)}\right),
\end{aligned}
$$

which is a polynomial in $\mathbf{q}$ of degree 1 , is a particular solution to the Schrödinger system (1.1). Here $\Delta$ is a twist cycle and $\varphi_{0}(t), \varphi_{n}^{(i)}(t)$ are certain rational $(L-1)$ forms defined by (4.1) and (4.2).

In order to generalize the hypergeometric integral (1.2) for particular solutions to the case of polynomials in $\mathbf{q}$ of degree $M \in \mathbb{Z}_{\geq 2}$, let us recall the equivalence between the Knizhnik-Zamolodchikov equation in conformal field theory and a quantization of a Schlesinger system [5], [12]. The KZ equations for the simple Lie algebra $\mathfrak{g}$ have integral representations of solutions taking values in tensor products of Verma modules of $\mathfrak{g}$ (see, for example, [1], [13]). From the point of view that the integral representation (1.2) may give a solution to the KZ equation, one should 
view the integral variables as corresponding to the simple roots of $\mathfrak{s l}_{L}$. For the case of $L=2$ and $N=1$, it is known that the Schrödinger system (1.1), the quantum sixth Painlevé equation, has hypergeometric solutions [9]:

$$
\begin{array}{r}
\int_{\Delta} \prod_{1 \leq a<b \leq M}\left(t^{(a)}-t^{(b)}\right)^{2 / \kappa} \prod_{a=1}^{M}\left(t^{(a)}\right)^{\alpha / \kappa}\left(1-z t^{(a)}\right)^{-\beta / \kappa}\left(1-t^{(a)}\right)^{-\gamma / \kappa} \\
\times\left(\varphi_{0}\left(t^{(a)}\right)-\varphi_{1}^{(1)}\left(t^{(a)}\right) q_{1}^{(1)}\right) .
\end{array}
$$

Note that the integrand above consists of $M$-copies of the integrand of (1.2) multiplied by the coupled term $\prod_{1 \leq a<b \leq M}\left(t^{(a)}-t^{(b)}\right)^{2 / \kappa}$.

Considering the above, we arrive at

Theorem 1.2 (see Theorem 4.3). The hypergeometric integral

$$
\begin{aligned}
\int_{\Delta} \prod_{\substack{1 \leq a<b \leq M \\
1 \leq n \leq L-1}}\left(t_{n}^{(a)}-t_{n}^{(b)}\right)^{2 / \kappa} \prod_{\substack{1 \leq a, b \leq M \\
1 \leq n \leq L-2}}\left(t_{n}^{(a)}-t_{n+1}^{(b)}\right)^{-1 / \kappa} \\
\times \prod_{a=1}^{M}\left\{\prod_{n=1}^{L-1}\left(t_{n}^{(a)}\right)^{\alpha_{n} / \kappa} \prod_{i=1}^{N}\left(1-z_{i} t_{L-1}^{(a)}\right)^{-\beta_{i} / \kappa}\left(1-t_{1}^{(a)}\right)^{-\gamma / \kappa}\right. \\
\left.\quad \times\left(\varphi_{0}\left(t^{(a)}\right)-\sum_{i=1}^{N} \sum_{n=1}^{L-1} \varphi_{n}^{(i)}\left(t^{(a)}\right) q_{n}^{(i)}\right)\right\},
\end{aligned}
$$

which is a polynomial in $\mathbf{q}$ of degree $M$, is a particular solution to the Schrödinger system (1.1). Here $\Delta$ is a skew-symmetric twist cycle.

The remainder of this paper is organized as follows. In Section 2, we introduce quantizations of the classical Hamiltonians of $\mathcal{H}_{L, N}$ and show that those quantum Hamiltonians are mutually commutative. In Section 3, we introduce our Schrödinger systems and discuss their properties. In Section 4, we give integral formulas for solutions.

Remark 1.3. As mentioned above, the classical Hamiltonian system $\mathcal{H}_{L, N}$ describes isomonodromic deformations for an $L \times L$ Fuchsian system

$$
\frac{\partial}{\partial u} \Phi(u)=\sum_{i=0}^{N+1} \frac{A_{i}}{u-u_{i}} \Phi(u),
$$

where $u_{0}=1, u_{i}=1 / z_{i}(1 \leq i \leq N)$, and $u_{N+1}=0$, whose spectral type is given by the $(N+3)$-tuple

$$
(1,1, \ldots, 1),(1,1, \ldots, 1),(L-1,1), \ldots,(L-1,1)
$$

of partitions of $L$. The spectral type defines multiplicities of the eigenvalues of 
each residue matrix $A_{i}$. Consequently, $L-1$ parameters are associated with the singular points 0 and $\infty$, and one parameter is associated with each singular point $u_{i}$ for $i=0, \ldots, N$. Notice that in the integrand given in the theorems above, $L-1$ parameters are associated with the singular point 0 , and one parameter is associated with each singular point $1,1 / z_{i}(1 \leq i \leq N)$.

\section{§2. Hamiltonian}

Let us define a non-commutative associative algebra $W_{L, N}$ over $\mathbb{C}$ with generators

$$
\begin{array}{ll}
q_{m}^{(i)}, p_{m}^{(i)} & (1 \leq m \leq L-1,1 \leq i \leq N), \\
e_{n}, \kappa_{n}, \theta_{j}, \hbar & (0 \leq n \leq L-1,0 \leq j \leq N)
\end{array}
$$

and commutation relations

$$
\left[p_{m}^{(j)}, q_{n}^{(i)}\right]=\delta_{n, m} \delta_{i, j} \hbar \quad(1 \leq n, m \leq L-1,1 \leq i, j \leq N),
$$

where $\delta_{i, j}$ is Kronecker's delta, and the other commutation relations are zero, and relations

$$
\sum_{m=0}^{L-1} e_{m}=\frac{L-1}{2}, \quad \sum_{m=0}^{L-1} \kappa_{m}=\sum_{i=0}^{N} \theta_{i} .
$$

The non-commutative associative algebra $W_{L, N}$ is an Ore domain, so that we can define its skew field $\mathcal{K}_{L, N}$ (see, for example, [2, Chapter 1, Section 8]).

Definition 2.1. We introduce Hamiltonians $H_{i}(i=1, \ldots, N)$ in the rational function field $W_{L, N}\left(z_{1}, \ldots, z_{N}\right)$ in variables $z_{1}, \ldots, z_{N}$ by

$$
\begin{aligned}
& z_{i} H_{i} \\
= & \sum_{n=0}^{L-1} e_{n} q_{n}^{(i)} p_{n}^{(i)}+\sum_{j=0}^{N} \sum_{0 \leq m<n \leq L-1} q_{m}^{(i)} p_{m}^{(j)} q_{n}^{(j)} p_{n}^{(i)}+\frac{1}{z_{i}-1} \sum_{m, n=0}^{L-1} q_{m}^{(i)} p_{m}^{(0)} q_{n}^{(0)} p_{n}^{(i)} \\
& +\sum_{\substack{j=1 \\
j \neq i}}^{N} \frac{z_{j}}{z_{i}-z_{j}} \sum_{m, n=0}^{L-1} q_{m}^{(i)} p_{n}^{(i)} q_{n}^{(j)} p_{m}^{(j)}+\theta_{i}\left(e_{0}+\kappa_{0}-\sum_{j=1}^{N} \theta_{j}-\sum_{\substack{j=1 \\
j \neq i}}^{N} \frac{\theta_{j} z_{j}}{z_{i}-z_{j}}\right),
\end{aligned}
$$

where

$$
\begin{array}{ll}
q_{0}^{(i)}=\theta_{i}+\sum_{m=1}^{L-1} q_{m}^{(i)} p_{m}^{(i)}, \quad p_{0}^{(i)}=-1 & (1 \leq i \leq N), \\
q_{m}^{(0)}=-1, \quad p_{m}^{(0)}=\kappa_{m}+\sum_{i=1}^{N} q_{m}^{(i)} p_{m}^{(i)} & (1 \leq m \leq L-1), \\
q_{0}^{(0)}=\kappa_{0}-\sum_{i=1}^{N} \theta_{i}-\sum_{i=1}^{N} \sum_{m=1}^{L-1} q_{m}^{(i)} p_{m}^{(i)}, \quad p_{0}^{(0)}=-1 .
\end{array}
$$


The Hamiltonians $H_{i}(i=1, \ldots, N)$ are the canonical quantizations of the polynomial Hamiltonians in [18, Appendix A]. By canonical quantization we mean replacing the Poisson bracket with the commutator.

Since the canonical variables in the classical Hamiltonians are not separated, quantization of the Hamiltonians is not unique. In the following, we show that the Hamiltonians $H_{i}$ are mutually commutative and the Schrödinger equations associated with the Hamiltonians $H_{i}$ have integral formulas.

Example 2.2. We give an example of the Hamiltonian $H_{i}$ in the case of $L=2$. Set $\left(q_{i}, p_{i}\right)=\left(q_{1}^{(i)}, p_{1}^{(i)}\right)$. The Hamiltonian $H_{i}$ is expressed as follows:

$$
\begin{aligned}
z_{i}\left(z_{i}-1\right) H_{i}= & q_{i}\left(\kappa_{1}-\theta_{0}+\sum_{j=1}^{N} q_{j} p_{j}\right)\left(\kappa_{1}+\sum_{j=1}^{N} q_{j} p_{j}\right)+z_{i}\left(\theta_{i}+q_{i} p_{i}\right) p_{i} \\
& -\sum_{\substack{j=1 \\
j \neq i}}^{N} \frac{z_{j}}{z_{i}-z_{j}}\left(\theta_{j}+q_{j} p_{j}\right) q_{i} p_{j}-\sum_{\substack{j=1 \\
j \neq i}}^{N} \frac{z_{i}}{z_{i}-z_{j}}\left(\theta_{i}+q_{i} p_{i}\right) q_{j} p_{i} \\
& -\sum_{\substack{j=1 \\
j \neq i}}^{N} \frac{z_{i}\left(z_{j}-1\right)}{z_{j}-z_{i}}\left(\theta_{i}+q_{i} p_{i}\right) q_{j} p_{j}-\sum_{\substack{j=1 \\
j \neq i}}^{N} \frac{z_{i}\left(z_{j}-1\right)}{z_{j}-z_{i}}\left(\theta_{j}+q_{j} p_{j}\right) q_{i} p_{i} \\
& -\left(z_{i}+1\right)\left(\theta_{i}+q_{i} p_{i}\right) q_{i} p_{i} \\
& -\left(\left(e_{1}-e_{0}\right) z_{i}+e_{0}-e_{1}-\hbar+\kappa_{1}-\kappa_{0}\right) q_{i} p_{i}
\end{aligned}
$$

plus some function of $\left(z_{1}, \ldots, z_{N}\right)$ only. These Hamiltonians are quantizations of the polynomial Hamiltonians for the Garnier system [6].

Example 2.3. We give an example of the Hamiltonian $H_{1}$ in the case of $N=1$. Set $\left(q_{m}, p_{m}\right)=\left(q_{m}^{(1)}, p_{m}^{(1)}\right), H=H_{1}$, and $z=z_{1}$. The Hamiltonian $H$ is written in a coupled form as follows:

$$
\begin{array}{r}
z(z-1) H=\sum_{m=1}^{L-1} H_{\mathrm{VI}}\left(\sum_{n=0}^{L-1} \alpha_{2 n+1}-\alpha_{2 m-1}-\eta, \sum_{n=0}^{m-1} \alpha_{2 n}, \sum_{n=m}^{L-1} \alpha_{2 n}, \alpha_{2 n-1} \eta ; q_{m}, p_{m}\right) \\
+\frac{1}{4} \sum_{1 \leq m<n \leq L-1}\left\{\left(\left(q_{m}-1\right) p_{m} q_{m}+q_{m} p_{m}\left(q_{m}-1\right)+2 \alpha_{2 m-1}\left(q_{m}-1\right)\right)\right. \\
\quad \times\left(p_{n}\left(q_{n}-z\right)+\left(q_{n}-z\right) p_{n}\right) \\
+\left(\left(q_{n}-z\right) p_{n} q_{n}+q_{n} p_{n}\left(q_{n}-z\right)+2 \alpha_{2 n-1}\left(q_{n}-z\right)\right) \\
\left.\times\left(p_{m}\left(q_{m}-1\right)+\left(q_{m}-1\right) p_{m}\right)\right\}
\end{array}
$$

plus some function of $\left(z_{1}, \ldots, z_{N}\right)$ only, where 


$$
\begin{aligned}
H_{\mathrm{VI}}\left(a_{0}, a_{1},\right. & \left.a_{z}, a ; q, p\right) \\
= & \frac{1}{6}(q p(q-1) p(q-z)+(q-1) p(q-z) p q+(q-z) p q p(q-1) \\
& +(q-z) p(q-1) p q+(q-1) p q p(q-z)+q p(q-z) p(q-1)) \\
& -\frac{1}{2}\left(a_{0}((q-1) p(q-z)+(q-z) p(q-1))+a_{1}(q p(q-z)+(q-z) p q)\right. \\
& \left.+\left(a_{z}-1\right)(q p(q-1)+(q-1) p q)\right)+a q .
\end{aligned}
$$

Here,

$$
\begin{array}{ll}
\alpha_{2 m-1}=\kappa_{n}-\hbar & (1 \leq m \leq L-1), \\
\alpha_{2 m}=e_{m}-e_{m+1}-\kappa_{m}+\hbar & (1 \leq m \leq L-2), \\
\alpha_{0}=e_{0}-e_{1}, \quad \alpha_{2 L-1}=-\kappa_{0}+(L-2) \hbar, \quad \sum_{m=0}^{2 L-1} \alpha_{m}=\kappa, \\
\eta=-\kappa_{0}+\theta_{1}-\frac{L-2}{2} \hbar .
\end{array}
$$

$H_{\mathrm{VI}}$ is the Hamiltonian of the quantum sixth Painlevé equation with affine Weyl group symmetry of type $D_{4}^{(1)}$ introduced in [8]. The affine Weyl group symmetry of type $D_{4}^{(1)}$ enables us to construct integral representations of particular solutions of the quantum sixth Painleve equation [10].

The Hamiltonian $H$ is a quantization of the Hamiltonian obtained by K. Fuji and T. Suzuki [3] $(L=3)$ and T. Suzuki [14] $(L \geq 3)$. The classical Hamiltonian system associated with $H$ has affine Weyl group symmetry of type $A_{2 L-1}^{(1)}$, as Bäcklund transformations. Quantization of this symmetry as in [7], [8] will be discussed in the near future.

\section{$\S 2.1$. Commutativity}

The Hamiltonians $H_{i}(i=1, \ldots, N)$ are expressed as follows:

$$
-z_{i}^{2} H_{i}=\sum_{\substack{j=0 \\ j \neq i}}^{N+1} \frac{\Omega_{i, j}}{u_{i}-u_{j}}-z_{i} C,
$$

where $\Omega_{i, j}, C$ are elements in $W_{L, N}$ and $C$ is a center, and $u_{0}=1, u_{i}=1 / z_{i}$ $(i=1, \ldots, n)$ and $u_{N+1}=0$.

For $i, j=1, \ldots, N$, the forms $\Omega_{i, j}$ are given by

$$
\Omega_{i, j}=\operatorname{tr}\left(\widehat{A}^{(i)} \widehat{A}^{(j)}\right),
$$

where $\widehat{A}^{(i)}$ is an $L \times L$ matrix defined as

$$
\left(\widehat{A}^{(i)}\right)_{m, n}=q_{m}^{(i)} p_{n}^{(i)}
$$


for $m, n=0,1, \ldots, L-1$, where $\left(\widehat{A}^{(i)}\right)_{m, n}$ is the $(m, n)$ entry of the matrix $\widehat{A}^{(i)}$. The entries of $\widehat{A}^{(i)}$ satisfy the following commutation relations.

Lemma 2.4. We have

$$
\frac{1}{\hbar}\left[\left(\widehat{A}^{i}\right)_{m, n},\left(\widehat{A}^{j}\right)_{m^{\prime}, n^{\prime}}\right]=\delta_{i, j}\left(\delta_{n, m^{\prime}}\left(\widehat{A}^{i}\right)_{m, n^{\prime}}-\delta_{n^{\prime}, m}\left(\widehat{A}^{i}\right)_{m^{\prime}, n}\right)
$$

for $0 \leq m, n, m^{\prime}, n^{\prime} \leq L-1$ and $1 \leq i, j \leq N$.

The proof is by straightforward calculation.

Recall the definition of Gaudin Hamiltonians (see, for example, [5, Section 2]). The Gaudin Hamiltonians $G_{i}(i=1, \ldots, N)$ for $\mathfrak{g l}_{L}$ are defined as

$$
G_{i}=\sum_{\substack{j=1 \\ j \neq i}}^{N} \frac{\operatorname{tr}\left(B^{(i)} B^{(j)}\right)}{u_{i}-u_{j}},
$$

where $B^{(i)}(i=1, \ldots, N)$ are $L \times L$ matrices whose entries satisfy the commutation relations (2.5). Since the commutativity of the Gaudin Hamiltonians amounts to the so-called infinitesimal braid relations, Lemma 2.4 yields

$$
\begin{array}{ll}
{\left[\Omega_{i, j}, \Omega_{k, l}\right]=0} & (i, j, k, l \text { distinct }), \\
{\left[\Omega_{i, j}, \Omega_{i, k}+\Omega_{k, j}\right]=0} & (i, j, k \text { distinct })
\end{array}
$$

for $i, j, k, l=1, \ldots, N$.

The other elements $\Omega_{i, 0}$ and $\Omega_{i, N+1}(i=1, \ldots, N)$ cannot be expressed in a similar way to (2.3) and (2.4). However, we can check by straightforward calculation that the infinitesimal braid relations above hold even if $i, j, k, l=$ $0,1, \ldots, N+1$. Therefore, we have

Proposition 2.5. The Hamiltonians $H_{i}(i=1, \ldots, N)$ are mutually commutative.

The proof is omitted since the calculation is lengthy.

\section{§3. Schrödinger system}

Denote by

$$
H_{i}\left(\mathbf{q}, \frac{\partial}{\partial \mathbf{q}}, \mathbf{z}\right)
$$

for $i=1, \ldots, N$ the Hamiltonians obtained by identifying $q_{m}^{(i)}$ and $p_{m}^{(i)}$ with $q_{m}^{(i)}$ and $\partial / \partial q_{m}^{(i)}$, respectively, of the Hamiltonians $H_{i}$ defined in Definition 2.1. 
We consider the following Schrödinger system:

$$
\kappa \frac{\partial}{\partial z_{i}} \Psi(\mathbf{q}, \mathbf{z})=H_{i}\left(\mathbf{q}, \frac{\partial}{\partial \mathbf{q}}, \mathbf{z}\right) \Psi(\mathbf{q}, \mathbf{z}),
$$

where $\kappa \in \mathbb{C}$, and $\Psi(\mathbf{q}, \mathbf{z})$ is an unknown function of

$$
\mathbf{q}=\left(q_{1}^{(1)}, \ldots, q_{L-1}^{(1)}, q_{1}^{(2)}, \ldots, q_{L-1}^{(2)}, \ldots, q_{1}^{(N)}, \ldots, q_{L-1}^{(N)}\right)
$$

and of $\mathbf{z}=\left(z_{1}, \ldots, z_{N}\right)$. Here, we regard $e_{n}, \kappa_{n}, \theta_{i}$ as complex parameters.

Proposition 3.1. The Schrödinger system (3.1) is completely integrable in the sense of Frobenius, that is,

for $i, j=1, \ldots, N$.

$$
\left[\kappa \frac{\partial}{\partial z_{i}}-H_{i}\left(\mathbf{q}, \frac{\partial}{\partial \mathbf{q}}, \mathbf{z}\right), \kappa \frac{\partial}{\partial z_{j}}-H_{j}\left(\mathbf{q}, \frac{\partial}{\partial \mathbf{q}}, \mathbf{z}\right)\right]=0
$$

Proof. Thanks to Proposition 2.5, we have only to show

$$
\frac{\partial}{\partial z_{i}} H_{j}\left(\mathbf{q}, \frac{\partial}{\partial \mathbf{q}}, \mathbf{z}\right)=\frac{\partial}{\partial z_{j}} H_{i}\left(\mathbf{q}, \frac{\partial}{\partial \mathbf{q}}, \mathbf{z}\right) .
$$

This is easily calculated as follows. For $i \neq j$, we have

$$
\begin{aligned}
\frac{\partial}{\partial z_{i}} H_{j}\left(\mathbf{q}, \frac{\partial}{\partial \mathbf{q}}, \mathbf{z}\right) & =\frac{\partial}{\partial z_{i}}\left(\frac{z_{i}}{z_{j}\left(z_{j}-z_{i}\right)}\left(\sum_{m, n=0}^{L-1} q_{m}^{(j)} p_{n}^{(j)} q_{n}^{(i)} p_{m}^{(i)}-\theta_{i} \theta_{j}\right)\right) \\
& =\frac{1}{\left(z_{i}-z_{j}\right)^{2}}\left(\sum_{m, n=0}^{L-1} q_{m}^{(j)} p_{n}^{(j)} q_{n}^{(i)} p_{m}^{(i)}-\theta_{i} \theta_{j}\right) .
\end{aligned}
$$

From Lemma 2.4, the last line is symmetrical with respect to $i$ and $j$, which finishes the proof.

In the simplest case, namely, the case of $L=2$ and $N=1$, the Schrödinger system (3.1) is the quantum sixth Painlevé equation. In the previous work [9], we showed that the quantum sixth Painlevé equation has polynomial solutions in terms of $q$.

In the general case, the Schrödinger system (3.1) also has polynomial solutions in terms of $\mathbf{q}$ due to the following propositions.

For an $L-1 \times N$ matrix $A$ whose entries are non-negative integers, let $q^{A}$ be the monomial defined by

$$
q^{A}=\prod_{m=1}^{L-1} \prod_{i=1}^{N}\left(q_{m}^{(i)}\right)^{A_{m, i}}
$$

where $A_{m, i}$ is the $(m, i)$ entry of the matrix $A$. Set $d(A)=\sum_{m=1}^{L-1} \sum_{i=1}^{N} A_{m, i}$. 
Proposition 3.2. The Hamiltonians $H_{i}(\mathbf{q}, \partial / \partial \mathbf{q}, \mathbf{z})(i=1, \ldots, N)$ act on $q^{A}$ as follows:

$$
\begin{aligned}
& z_{i} H_{i}\left(q^{A}\right)=\left\{\sum_{n=1}^{L-1} A_{n, i}\left(e_{n}-e_{0}-\theta_{i}-\sum_{m=1}^{n} A_{m, i}\right)+\theta_{i}\left(\kappa_{0}-\sum_{j=1}^{N} \theta_{j}\right)\right. \\
& +\frac{1}{z_{i}-1}\left(\theta_{i}+\sum_{n=1}^{L-1} A_{n, i}\right)\left(\kappa_{0}-\sum_{j=1}^{N} \theta_{j}-\sum_{j=1}^{N} \sum_{n=1}^{L-1} A_{n, j}\right) \\
& -\frac{1}{z_{i}-1} \sum_{n=1}^{L-1} A_{n, i}\left(\kappa_{n}+\sum_{j=1}^{N} A_{n, j}-1\right) \\
& \left.+\sum_{\substack{j=1 \\
j \neq i}} \frac{z_{j}}{z_{i}-z_{j}} \sum_{n=1}^{L-1}\left(A_{n, i}\left(\sum_{m=1}^{L-1} A_{m, j}+A_{n, j}+\theta_{j}\right)+\theta_{i} A_{n, j}\right)\right\} q^{A} \\
& -\frac{1}{z_{i}-1}\left(\kappa_{0}-\sum_{j=1}^{N} \theta_{j}-\sum_{j=1}^{N} \sum_{m=1}^{L-1} A_{m, j}\right) \sum_{n=1}^{L-1}\left(\kappa_{n}+\sum_{j=1}^{N} A_{n, j}\right) q^{\left(A_{n, i}+1\right)} \\
& +\frac{z_{i}}{z_{i}-1}\left(\theta_{i}+\sum_{m=1}^{L-1} A_{m, i}-1\right) \sum_{n=1}^{L-1} A_{n, i} q^{\left(A_{n, i}-1\right)} \\
& -\frac{1}{z_{i}-1} \sum_{n=1}^{L-1}\left(\kappa_{n}+\sum_{j=1}^{N} A_{n, j}\right)\left(\sum_{m=1}^{n-1} A_{m, i} q^{\left(A_{n, i}+1, A_{m, i}-1\right)}\right. \\
& \left.+z_{i} \sum_{m=n+1}^{L-1} A_{m, i} q^{\left(A_{n, i}+1, A_{m, i}-1\right)}\right) \\
& +\sum_{\substack{j=1 \\
j \neq i}}^{N} \frac{1}{z_{i}-z_{j}} \sum_{n=1}^{L-1} A_{n, j}\left(z_{j} \sum_{m=1}^{n-1} A_{m, i} q^{\left(A_{m, j}+1, A_{m, i}-1, A_{n, i}+1, A_{n, j}-1\right)}\right. \\
& \left.+z_{i} \sum_{m=1}^{n-1} A_{m, i} q^{\left(A_{m, j}+1, A_{m, i}-1, A_{n, i}+1, A_{n, j}-1\right)}\right) \\
& -\sum_{\substack{j=1 \\
j \neq i}}^{N} \frac{z_{i}}{z_{i}-z_{j}}\left(\theta_{i}+\sum_{m=1}^{L-1} A_{m, i}-1\right) \sum_{n=1}^{L-1} A_{n, i} q^{\left(A_{n, j}+1, A_{n, i}-1\right)} \\
& -\sum_{\substack{j=1 \\
j \neq i}}^{N} \frac{z_{j}}{z_{i}-z_{j}}\left(\theta_{j}+\sum_{m=1}^{L-1} A_{m, j}-1\right) \sum_{n=1}^{L-1} A_{n, j} q^{\left(A_{n, i}+1, A_{n, j}-1\right)}
\end{aligned}
$$

where

$$
q^{\left(A_{n, i}+1\right)}=q^{A} q_{n}^{(i)}, \quad q^{\left(A_{n, i}-1\right)}=\frac{q^{A}}{q_{n}^{(i)}}, \quad q^{\left(A_{n, i}+1, A_{m, j}-1\right)}=\frac{q^{A} q_{n}^{(i)}}{q_{m}^{(j)}}
$$




$$
q^{\left(A_{m, j}+1, A_{m, i}-1, A_{n, i}+1, A_{n, j}-1\right)}=\frac{q^{A} q_{m}^{(j)} q_{n}^{(i)}}{q_{m}^{(i)} q_{n}^{(j)}}
$$

for $1 \leq n, m \leq L-1,1 \leq i, j \leq N$

Proof. Note that the Hamiltonian $H_{i}$ defined in (2.2) consists of five terms. The actions of the first and last terms on $q^{A}$ are trivial. The action of the second term divided by $z_{i}$ on $q^{A}$ is computed as follows. First, divide it into four parts:

$$
\begin{aligned}
\sum_{j=0}^{N} \sum_{0 \leq m<n \leq L-1} q_{m}^{(i)} p_{m}^{(j)} & q_{n}^{(j)} p_{n}^{(i)}=\sum_{j=1}^{N} \sum_{1 \leq m<n \leq L-1} q_{m}^{(i)} p_{m}^{(j)} q_{n}^{(j)} p_{n}^{(i)} \\
& -\sum_{j=1}^{N} \sum_{n=1}^{L-1} q_{0}^{(i)} q_{n}^{(j)} p_{n}^{(i)}-\sum_{1 \leq m<n \leq L-1} q_{m}^{(i)} p_{m}^{(0)} p_{n}^{(i)}+\sum_{n=1}^{L-1} q_{0}^{(i)} p_{n}^{(i)}
\end{aligned}
$$

By Definition 2.1, we have

$$
\begin{aligned}
\sum_{j=0}^{N} \sum_{0 \leq m<n \leq L-1} q_{m}^{(i)} p_{m}^{(j)} q_{n}^{(j)} p_{n}^{(i)}\left(q^{A}\right) & \\
= & \sum_{j=1}^{N} \sum_{1 \leq m<n \leq L-1} A_{m, j} A_{n, i} q^{\left(A_{n, j}+1, A_{n, i}-1, A_{m, i}+1, A_{m, j}-1\right)} \\
& -\sum_{j=1}^{N}\left(\theta_{i}+\sum_{m=1}^{L-1} A_{m, i}-1\right) \sum_{n=1}^{L-1} A_{n, i} q^{\left(A_{n, j}+1, A_{n, i}-1\right)} \\
& -\sum_{1 \leq m<n \leq L-1}\left(\kappa_{m}+\sum_{j=1}^{N} A_{m, j}\right) A_{n, i} q^{\left(A_{m, i}+1, A_{n, i}-1\right)} \\
& +\left(\theta_{i}+\sum_{m=1}^{L-1} A_{m, i}-1\right) \sum_{n=1}^{L-1} A_{n, i} q^{\left(A_{n, i}-1\right)}
\end{aligned}
$$

We omit the calculation of the actions of the third and fourth terms on $q^{A}$ since they are computed similarly. Summing up the results of the actions of all terms on $q^{A}$, we obtain (3.2).

Let $V(M)\left(M \in \mathbb{Z}_{\geq 0}\right)$ be the subspace of the polynomial ring $\mathbb{C}[\mathbf{q}]$ defined by $V(M)=\bigoplus_{A} \mathbb{C} q^{A}$, where the summation is taken over all $L-1 \times N$ matrices $A$ such that $d(A) \leq M$.

Proposition 3.3. For each $i=1, \ldots, N$, the Hamiltonian $H_{i}(\mathbf{q}, \partial / \partial \mathbf{q}, \mathbf{z})$ acts on $V(M)$ if $\kappa_{0}-\sum_{i=1}^{N} \theta_{i}=M$.

Proof. We compute the action of the Hamiltonian $H_{i}(\mathbf{q}, \partial / \partial \mathbf{q}, \mathbf{z})$ on $q^{A}$ such that $d(A)=M$ as follows. From Proposition 3.2, we have 


$$
\begin{aligned}
z_{i}\left(z_{i}-1\right) & H_{i}\left(\mathbf{q}, \frac{\partial}{\partial \mathbf{q}}, \mathbf{z}\right)\left(q^{A}\right) \\
& =-\left(\kappa_{0}-\sum_{i=1}^{N} \theta_{i}-M\right) \sum_{n=1}^{L-1}\left(\kappa_{n}+\sum_{j=1}^{N} A_{n, j}\right) q_{n}^{(i)} q^{A}+f(\mathbf{q}) .
\end{aligned}
$$

Here $f(\mathbf{q})$ is a polynomial of degree at most $M$. Hence, if $\kappa_{0}-\sum_{i=1}^{N} \theta_{i}=M$, then the first term of (3.3) vanishes, which finishes the proof.

By Proposition 3.3, for the Schrödinger equation (3.1), we can consider polynomial solutions

$$
\Psi(\mathbf{q}, \mathbf{z})=\sum_{A \in \mathcal{A}_{M}} c_{A}(\mathbf{z}) q^{A},
$$

where

$$
\mathcal{A}_{M}=\left\{A=\left(A_{m, i}\right) \mid A_{m, i} \in \mathbb{Z}_{\geq 0}, d(A) \leq M\right\}
$$

and $c_{A}(\mathbf{z})$ is a function of $\mathbf{z}$. In the next section, we present integral formulas for polynomial solutions taking values in $V(M)$.

The Hamiltonians $H_{i}$ also act on other subspaces of the polynomial ring $\mathbb{C}[\mathbf{q}]$. Let $F\left(T_{1}, \ldots, T_{L-1}\right)\left(T_{1}, \ldots, T_{L-1} \in \mathbb{Z}_{\geq 0}\right)$ be the subspace of the polynomial ring $\mathbb{C}[\mathbf{q}]$ defined as $F\left(T_{1}, \ldots, T_{L-1}\right)=\bigoplus_{A} \mathbb{C} q^{A}$, where the summation is taken over all $L-1 \times N$ matrices $A$ such that the entries of $A$ are non-negative integers and $\sum_{i=1}^{N} A_{n, i} \leq T_{n}(n=1, \ldots, L-1)$. Set $d_{n}(A)=\sum_{i=1}^{N} A_{n, i}$.

Proposition 3.4. For each $i=1, \ldots, N$, the Hamiltonian $H_{i}(\mathbf{q}, \partial / \partial \mathbf{q}, \mathbf{z})$ acts on $F\left(T_{1}, \ldots, T_{L-1}\right)$ if $\kappa_{n}=-T_{n}$ for $n=1, \ldots, L-1$.

Proof. Take an $L-1 \times N$ matrix $A$ such that the entries of $A$ are non-negative integers and $d_{n}(A)=T_{n}$ for any $n \in\{1, \ldots, L-1\}$. We compute the action of the Hamiltonian $H_{i}(\mathbf{q}, \partial / \partial \mathbf{q}, \mathbf{z})$ on $q^{A}$ as follows. From Proposition 3.2, we have

$$
\begin{aligned}
& z_{i}\left(1-z_{i}\right) H_{i}\left(\mathbf{q}, \frac{\partial}{\partial \mathbf{q}}, \mathbf{z}\right)\left(q^{A}\right) \\
= & \left(\kappa_{0}-\sum_{j=1}^{N} \theta_{j}-\sum_{m=1}^{L-1} T_{m}\right) \sum_{n=1}^{L-1}\left(\kappa_{n}+T_{n}\right) q^{\left(A_{n, i}+1\right)} \\
& +\sum_{n=1}^{L-1}\left(\kappa_{n}+T_{n}\right)\left(\sum_{m=1}^{n-1} A_{m, i} q^{\left(A_{n, i}+1, A_{m, i}-1\right)}+z_{i} \sum_{m=n+1}^{L-1} A_{m, i} q^{\left(A_{n, i}+1, A_{m, i}-1\right)}\right) \\
& +f(\mathbf{q}) .
\end{aligned}
$$

Here, $f(\mathbf{q})$ is a polynomial such that for each $n \in\{1, \ldots, L-1\}$, the degree of $f(\mathbf{q})$ as a polynomial in $q_{n}^{(1)}, \ldots, q_{n}^{(N)}$ is less than or equal to $d_{n}(A)$. Thus, if $\kappa_{n}=-T_{n}$ 
for $n=1, \ldots, L-1$, then the first and second terms of (3.5) vanish, which finishes the proof.

Consequently, we can also consider polynomial solutions taking values in $F\left(T_{1}, \ldots, T_{L-1}\right)$.

\section{$\S 4$. Integral formulas}

In this section, we construct integral formulas for polynomial solutions of the Schrödinger systems (3.1).

Recall that the Gauss hypergeometric function is a particular solution to both the classical and quantum sixth Painlevé equation [9]. Hypergeometric solutions to the classical Hamiltonian systems $\mathcal{H}_{L, N}$ were given by T. Suzuki [15] $(L \geq 2$, $N=1)$ and $\mathrm{T}$. Tsuda [17] $(L \geq 2, N \geq 1)$ independently, under the condition $\kappa_{0}-\sum_{i=1}^{N} \theta_{i}=0$.

These hypergeometric solutions are the generalized hypergeometric functions (Thomae's hypergeometric functions) ${ }_{L} F_{L-1}$ in the case of $(L \geq 2, N=1)$ and their generalizations in the case of $(L \geq 2, N \geq 1)$.

We expect that these generalized hypergeometric functions are also solutions to a quantization of the classical Hamiltonian systems $\mathcal{H}_{L, N}$, the Schrödinger systems (3.1). Indeed, this is true if we consider polynomial solutions to the Schrödinger systems (3.1) with $\kappa_{0}-\sum_{i=1}^{N} \theta_{i}=1$.

Set $\kappa_{0}-\sum_{i=1}^{N} \theta_{i}=M \in \mathbb{Z}_{\geq 0}$. We begin with the case $M=1$ and later we deal with the general case.

\section{$\S 4.1$. The case of $M=1$}

Consider the multivalued function

$$
U(t)=\prod_{n=1}^{L-1} t_{n}^{\alpha_{n} / \kappa} \prod_{i=1}^{N}\left(1-z_{i} t_{L-1}\right)^{-\beta_{i} / \kappa} \prod_{n=1}^{L-1}\left(t_{n-1}-t_{n}\right)^{-\gamma_{n} / \kappa}
$$

with $t_{0}=1$ defined on the complement $T \subset \mathbb{C}^{L-1}$ of the singular locus $D$ given by

$$
D=\bigcup_{1 \leq n \leq L-1}\left\{t_{n-1}=t_{n}\right\} \cup \bigcup_{1 \leq n \leq L-1}\left\{t_{n}=0\right\} \cup \bigcup_{1 \leq i \leq N}\left\{t_{L-1}=1 / z_{i}\right\} .
$$

Let $\mathcal{S}$ be the rank one local system determined by $U(t)$, and $\mathcal{S}^{*}$ the dual local system of $\mathcal{S}$. The hypergeometric pairing between the twisted homology group and the twisted de Rham cohomology group is

$$
H_{L-1}\left(T, \mathcal{S}^{*}\right) \times H^{L-1}(T, \nabla) \rightarrow \mathbb{C}, \quad(\Delta, \varphi) \mapsto \int_{\Delta} U(t) \varphi,
$$


where $\varphi$ is a rational $(L-1)$-form holomorphic outside $D$, and $\nabla$ is the covariant differential operator given by $\nabla=d+d \log (U(t)) \wedge$.

According to [17], the rational $(L-1)$-forms

$$
\begin{gathered}
\varphi_{0}(t)=\frac{d t_{1} \wedge \cdots \wedge d t_{L-1}}{t_{L-1}} \prod_{n=1}^{L-1} \frac{1}{t_{n-1}-t_{n}}, \\
\varphi_{n}^{(i)}(t)=\frac{d t_{1} \wedge \cdots \wedge d t_{L-1}}{\left(1-z_{i} t_{L-1}\right) t_{L-1}} \prod_{\substack{m=1 \\
m \neq n}}^{L-1} \frac{1}{t_{m-1}-t_{m}}
\end{gathered}
$$

represent a basis of $H^{L-1}(T, \nabla)$.

Define $\Psi_{1}(\mathbf{q}, \mathbf{z})$ by

$$
\Psi_{1}(\mathbf{q}, \mathbf{z})=\int_{\Delta} U(t)\left(\varphi_{0}(t)-\sum_{i=1}^{N} \sum_{n=1}^{L-1} \varphi_{n}^{(i)}(t) q_{n}^{(i)}\right)
$$

with $\Delta \in H_{L-1}\left(T, \mathcal{S}^{*}\right)$. From Proposition 3.3, when $\kappa_{0}-\sum_{i=1}^{N} \theta_{i}=1$, the action of the Hamiltonian $H_{i}(i=1, \ldots, N)$ on $\Psi_{1}, H_{i} \Psi_{1}(\mathbf{q}, \mathbf{z})$, is also a polynomial of degree $\leq 1$, and the constant term and the coefficient of $q_{n}^{(j)}(1 \leq n \leq L-1,1 \leq$ $j \leq N)$ of $H_{i} \Psi_{1}(\mathbf{q}, \mathbf{z})$ are linear combinations of $\int_{\Delta} U(t) \varphi_{0}(t)$ and $\int_{\Delta} U(t) \varphi_{n}^{(j)}(t)$ $(1 \leq n \leq L-1,1 \leq j \leq N)$. Remarkably they coincide with $\kappa \partial \varphi_{0}(t) / \partial z_{i}$ and $\kappa \partial \varphi_{n}^{(j)}(t) / \partial z_{i}$ with appropriate correspondence between parameters. Namely, we have

Theorem 4.1. If $\kappa_{0}-\sum_{i=1}^{N} \theta_{i}=1$, then $\Psi_{1}(\mathbf{q}, \mathbf{z})$ is a solution to the Schrödinger system (3.1) with

$$
\alpha_{n}=e_{n+1}-e_{n}+\kappa_{n+1}, \quad \beta_{i}=-\theta_{i}, \quad \gamma_{n}=\kappa_{n},
$$

for $1 \leq n \leq L-1$ and $1 \leq i \leq N$, where $e_{L}=e_{0}$ and $\kappa_{L}=1$.

\section{$\S 4.2$. The case of $M \geq 2$}

Fix $M \in \mathbb{Z}_{\geq 2}$. We consider the multivalued function

$$
\begin{aligned}
U(t)= & \prod_{\substack{1 \leq a<b \leq M, 1 \leq n \leq L-1}}\left(t_{n}^{(a)}-t_{n}^{(b)}\right)^{2 / \kappa} \prod_{\substack{1 \leq a, b \leq M \\
1 \leq n \leq L-2}}\left(t_{n}^{(a)}-t_{n+1}^{(b)}\right)^{-1 / \kappa} \\
& \times \prod_{a=1}^{M}\left\{\prod_{n=1}^{L-1}\left(t_{n}^{(a)}\right)^{\alpha_{n} / \kappa} \prod_{i=1}^{N}\left(1-z_{i} t_{L-1}^{(a)}\right)^{-\beta_{i} / \kappa}\left(1-t_{1}^{(a)}\right)^{-\gamma / \kappa}\right\}
\end{aligned}
$$


defined on the complement $T \subset \mathbb{C}^{(L-1) M}$ of the singular locus $D$ given by

$$
\begin{aligned}
D= & \bigcup_{\substack{1 \leq a<b \leq M \\
1 \leq n \leq L-1}}\left\{t_{n}^{(a)}=t_{n}^{(b)}\right\} \cup \bigcup_{\substack{1 \leq a, b \leq M \\
1 \leq n \leq L-2}}\left\{t_{n}^{(a)}=t_{n+1}^{(b)}\right\} \cup \bigcup_{\substack{1 \leq a \leq M \\
1 \leq n \leq L-1}}\left\{t_{n}^{(a)}=0\right\} \\
& \cup \bigcup_{\substack{1 \leq a \leq M \\
1 \leq i \leq N}}\left\{t_{L-1}^{(a)}=1 / z_{i}\right\} \cup \bigcup_{1 \leq a \leq M}\left\{t_{1}^{(a)}=1\right\} .
\end{aligned}
$$

Let $\mathcal{S}$ be the rank one local system determined by $U(t)$, and $\mathcal{S}^{*}$ the dual local system of $\mathcal{S}$. The hypergeometric pairing between the twisted homology group and the twisted de Rham cohomology group is

$$
H_{(L-1) M}\left(T, \mathcal{S}^{*}\right) \times H^{(L-1) M}(T, \nabla) \rightarrow \mathbb{C}, \quad(\Delta, \varphi) \mapsto \int_{\Delta} U(t) \varphi,
$$

where $\varphi$ is a rational $(L-1) M$-form holomorphic outside $D$, and $\nabla$ is the covariant differential operator given by $\nabla=d+d \log (U(t)) \wedge$.

Denote by $\mathfrak{S}_{M}^{L-1}$ the $(L-1)$-th product of the symmetric group of degree $M$. Let $\mathfrak{S}_{M}^{L-1}$ act on a rational function $f(t)$ of variables $t=\left(t_{1}^{(1)}, \ldots, t_{L-1}^{(1)}, \ldots\right.$, $\left.t_{1}^{(M)}, \ldots, t_{L-1}^{(M)}\right)$ by

$$
\sigma(f(t))=f\left(t_{1}^{\left(\sigma_{1}(1)\right)}, \ldots, t_{L-1}^{\left(\sigma_{L-1}(1)\right)}, \ldots, t_{1}^{\left(\sigma_{1}(M)\right)}, \ldots, t_{L-1}^{\left(\sigma_{L-1}(M)\right)}\right)
$$

for $\sigma=\left(\sigma_{1}, \ldots, \sigma_{L-1}\right) \in \mathfrak{S}_{M}^{L-1}$. Let $\operatorname{Sym}[f(t)]$ be the symmetrization of $f(t)$, given by $\operatorname{Sym}[f(t)]=\sum_{\sigma \in \mathfrak{S}_{M}^{L-1}} \sigma(f(t))$.

Definition 4.2. For $M \in \mathbb{Z}_{\geq 2}$, we define

$$
\Psi_{M}(\mathbf{q}, \mathbf{z})=\int_{\Delta} U(t) \cdot \operatorname{Sym}\left[\prod_{a=1}^{M} \frac{1}{t_{L-1}^{(a)}}\left(f_{0}\left(t^{(a)}\right)-\sum_{i=1}^{N} \sum_{n=1}^{L-1} f_{n}^{(i)}\left(t^{(a)}\right) q_{n}^{(i)}\right)\right] d t
$$

where $\Delta \in H_{(L-1) M}\left(T, \mathcal{S}^{*}\right)$ and

$$
\begin{aligned}
& f_{0}\left(t^{(a)}\right)=\prod_{m=1}^{L-1} \frac{1}{t_{m-1}^{(a)}-t_{m}^{(a)}}, \quad f_{n}^{(i)}\left(t^{(a)}\right)=\frac{1}{1-z_{i} t_{L-1}^{(a)}} \prod_{\substack{m=1 \\
m \neq n}}^{L-1} \frac{1}{t_{m-1}^{(a)}-t_{m}^{(a)}}, \quad t_{0}^{(a)}=1, \\
& d t=d t_{1}^{(1)} \wedge \cdots \wedge d t_{L-1}^{(1)} \wedge d t_{1}^{(2)} \wedge \cdots \wedge d t_{L-1}^{(2)} \wedge \cdots \wedge d t_{1}^{(M)} \wedge \cdots \wedge d t_{L-1}^{(M)} .
\end{aligned}
$$

Theorem 4.3. If $\kappa_{0}-\sum_{i=1}^{N} \theta_{i}=M$ and $\kappa_{n}=1(2 \leq n \leq L-1)$, then $\Psi_{M}(\mathbf{q}, \mathbf{z})$ is a solution to the Schrödinger system (3.1) with

$$
\alpha_{n}=e_{n+1}-e_{n}+1, \quad \beta_{i}=-\theta_{i}, \quad \gamma=\kappa_{1}+M-1,
$$

for $1 \leq n \leq L-1$ and $1 \leq i \leq N$, where $e_{L}=e_{0}$. 
Note that the hypergeometric integral $\Psi_{M}(\mathbf{q}, \mathbf{z})$ is also a polynomial in $V(M)$ defined in the previous section, namely, it can be expressed as

$$
\Psi_{M}(\mathbf{q}, \mathbf{z})=\sum_{A \in \mathcal{A}_{M}} q^{A} \int_{\Delta} U(t) \varphi_{A}(t)
$$

with some rational $(L-1) M$-forms $\varphi_{A}(t)$.

Because $\varphi_{A}(t)$ is the sum of the products of $\prod_{a=1}^{M} 1 / t_{L-1}^{(a)}$,

$$
f_{0}\left(t^{\left(a_{1}^{0}\right)}\right), \ldots, f_{0}\left(t^{\left(a_{A_{0}}^{0}\right)}\right),-f_{n}^{(i)}\left(t^{\left(a_{1}^{n, j}\right)}\right), \ldots,-f_{n}^{(i)}\left(t^{\left(a_{A_{n, i}}^{n, j}\right)}\right)
$$

for $a_{1}^{0}, a_{j}^{n, i}=1, \ldots, M$ such that $a_{i}^{0} \neq a_{j}^{n, i}$ and $a_{j}^{n, i} \neq a_{\ell}^{m, k}$ if $(n, i, j) \neq(m, k, \ell)$, since $q^{A}=\prod_{n=1}^{L-1} \prod_{i=1}^{N}\left(q_{n}^{(i)}\right)^{A_{n, i}}$, we have the following expression:

$$
\begin{aligned}
\varphi_{A}(t)=\operatorname{Sym}\left[(-1)^{M-A_{0}}\left(\begin{array}{c}
M \\
A
\end{array}\right) \prod_{i=1}^{N} \prod_{n=1}^{L-1}\right. & \prod_{a=S_{n-1}^{(i)}+1}^{S_{n}^{(i)}} f_{n}^{(i)}\left(t^{(a)}\right) \\
& \left.\times \prod_{a=M-A_{0}+1}^{M} f_{0}\left(t^{(a)}\right) \prod_{a=1}^{M} \frac{1}{t_{L-1}^{(a)}}\right] d t,
\end{aligned}
$$

where

$$
\begin{aligned}
& A_{0}=M-\sum_{\substack{1 \leq i \leq N, 1 \leq n \leq L-1}} A_{n, i}, \quad\left(\begin{array}{c}
M \\
A
\end{array}\right)=\frac{M !}{A_{0} ! \prod_{\substack{1 \leq i \leq N, 1 \\
1 \leq n \leq L-1}} A_{n, i} !}, \\
& S_{n}^{(i)}=\sum_{j=1}^{i-1} \sum_{m=1}^{L-1} A_{m, j}+\sum_{m=1}^{n} A_{m, i} .
\end{aligned}
$$

Here, we used the following relations:

$$
\begin{aligned}
\operatorname{Sym}\left[g\left(t^{(a)}, t^{(b)}\right) f_{n}^{(i)}\left(t^{(a)}\right) f_{m}^{(j)}\left(t^{(b)}\right)\right] & =\operatorname{Sym}\left[g\left(t^{(b)}, t^{(a)}\right) f_{n}^{(i)}\left(t^{(b)}\right) f_{m}^{(j)}\left(t^{(a)}\right)\right], \\
\operatorname{Sym}\left[g\left(t^{(a)}, t^{(b)}\right) f_{n}^{(i)}\left(t^{(a)}\right) f_{0}\left(t^{(b)}\right)\right] & =\operatorname{Sym}\left[g\left(t^{(b)}, t^{(a)}\right) f_{n}^{(i)}\left(t^{(b)}\right) f_{0}\left(t^{(a)}\right)\right]
\end{aligned}
$$

for $1 \leq n, m \leq L-1,1 \leq i, j \leq N, 1 \leq a, b \leq M$, and a rational function $g\left(t^{(a)}, t^{(b)}\right)$.

We introduce a linear operator $\nabla_{i}(i=1, \ldots, N)$ acting on $\varphi$ via

$$
\nabla_{i} \varphi=\frac{1}{U} \frac{\partial U}{\partial z_{i}} \varphi+\frac{\partial \varphi}{\partial z_{i}}
$$

since in general, for an $(L-1) M$-form $\varphi$,

$$
\frac{\partial}{\partial z_{i}} \int_{\Delta} U \varphi=\int_{\Delta} U\left(\frac{1}{U} \frac{\partial U}{\partial z_{i}} \varphi+\frac{\partial \varphi}{\partial z_{i}}\right)
$$

Let us explain our proof of Theorem 4.3 briefly. We compute $\kappa \nabla_{i} \varphi_{A}(t)$ and obtain the linear Pfaffian system for $\left\{\int_{\Delta} U \varphi_{A}(t) \mid A \in \mathcal{A}_{M}\right\}$. On the other hand, we compute the action of the Hamiltonians $H_{i}$ on $q^{A}$ and obtain the coefficient of 
$q^{A}$ in $H_{i} \Psi_{M}(\mathbf{q}, \mathbf{z})$ as a linear combination of elements of $\left\{\int_{\Delta} U \varphi_{A}(t) \mid A \in \mathcal{A}_{M}\right\}$. Finally, comparing both results, we obtain Theorem 4.3.

Proof of Theorem 4.3. Fix $i \in\{1, \ldots, N\}$ and $A \in \mathcal{A}_{M}$. We compute $\nabla_{i} \varphi_{A}(t)$ as follows. First,

$$
\kappa \nabla_{i} \varphi_{A}(t)=\operatorname{Sym}\left[\left(\beta_{i} \sum_{a=1}^{M} \frac{t_{L-1}^{(a)}}{1-z_{i} t_{L-1}^{(a)}}+\kappa \sum_{a=S_{0}^{(i)}+1}^{S_{L-1}^{(i)}} \frac{t_{L-1}^{(a)}}{1-z_{i} t_{L-1}^{(a)}}\right) \bar{\varphi}_{A}(t)\right] d t,
$$

where $\bar{\varphi}_{A}(t)$ is defined by

$$
\bar{\varphi}_{A}(t)=(-1)^{M-A_{0}}\left(\begin{array}{c}
M \\
A
\end{array}\right) \prod_{i=1}^{N} \prod_{n=1}^{L-1} \prod_{a=S_{n-1}^{(i)}+1}^{S_{n}^{(i)}} f_{n}^{(i)}\left(t^{(a)}\right) \prod_{a=M-A_{0}+1}^{M} f_{0}\left(t^{(a)}\right) \prod_{a=1}^{M} \frac{1}{t_{L-1}^{(a)}} .
$$

Using the relations (4.4) and (4.5), we obtain

$$
\begin{array}{r}
\kappa \nabla_{i} \varphi_{A}(t)=\operatorname{Sym}\left[\left(\beta_{i} \sum_{\substack{j=1 \\
j \neq i}}^{N} \sum_{n=1}^{L-1} \frac{A_{n, j} t_{L-1}^{\left(S_{n}^{(j)}\right)}}{1-z_{i} t_{L-1}^{\left(S_{n}^{(j)}\right)}}+\beta_{i} \frac{A_{0} t_{L-1}^{\left(M-A_{0}+1\right)}}{1-z_{i} t_{L-1}^{\left(M-A_{0}+1\right)}}\right.\right. \\
\left.\left.+\left(\beta_{i}+\kappa\right) \sum_{n=1}^{L-1} \frac{A_{n, i} t_{L-1}^{\left(S_{n}^{(i)}\right)}}{1-z_{i} t_{L-1}^{\left(S_{n}^{(i)}\right)}}\right) \bar{\varphi}_{A}(t)\right] d t .
\end{array}
$$

Since

$$
\frac{t}{1-z_{i} t}=\frac{1-z_{j} t}{z_{i}-z_{j}}\left(\frac{1}{1-z_{i} t}-\frac{1}{1-z_{j} t}\right),
$$

the first term of (4.6) equals

$$
\beta_{i} \sum_{\substack{j=1 \\ j \neq i}}^{N} \sum_{n=1}^{L-1} \frac{1}{z_{i}-z_{j}}\left(\left(A_{n, i}+1\right) \varphi_{\left(A_{n, j}-1, A_{n, i}+1\right)}(t)-A_{n, j} \varphi_{A}(t)\right),
$$

where $\varphi_{\left(A_{n, j}-1, A_{n, i}+1\right)}(t)$ is the rational $(L-1) M$-form defined for the matrix in $\mathcal{A}_{M}$ whose $(n, j)$ entry is $A_{n, j}-1$ and $(n, i)$ entry is $A_{n, i}+1$, and the other $(m, k)$ entries are $A_{m, k}$.

As for the second term of (4.6), since

$$
\frac{t_{L-1}}{1-z_{i} t_{L-1}}=\frac{1}{\left(z_{i}-1\right) f_{0}(t)}\left(-f_{0}(t)+\sum_{n=1}^{L-1} f_{n}^{(i)}(t)\right)
$$

the second term of (4.6) equals

$$
\frac{-\beta_{i}}{z_{i}-1}\left(A_{0} \varphi_{A}(t)+\sum_{n=1}^{L-1}\left(A_{n, i}+1\right) \varphi_{\left(A_{n, i}+1\right)}(t)\right)
$$


where $\varphi_{\left(A_{n, i}+1\right)}(t)$ is the rational $(L-1) M$-form defined for the matrix in $\mathcal{A}_{M}$ whose $(n, i)$ entry is $A_{n, i}+1$, and the other $(m, k)$ entries are $A_{m, k}$.

In order to calculate the third term of (4.6), we compute the coboundaries $X_{n}^{(i)}(n=1, \ldots, L-1)$ defined by

$$
X_{n}^{(i)}=\kappa \sum_{m=n}^{L-1} \nabla\left(\sum_{\sigma \in \mathfrak{S}_{M}^{L-1}} \sigma\left(t_{m}^{\left(S_{n}^{(i)}\right)} \bar{\varphi}_{A}(t)\right) * d t_{m}^{\left(\sigma_{m}\left(S_{n}^{(i)}\right)\right)}\right),
$$

where $* d t_{m}^{(a)}$ is defined by

$$
* d t_{m}^{(a)}=(-1)^{(L-1)(a-1)+m-1} d t_{1}^{(1)} \wedge \cdots \wedge \widehat{d t_{m}^{(a)}} \wedge \cdots \wedge t_{L-1}^{(M)},
$$

so that $d t_{m}^{(a)} \wedge * d t_{m}^{(a)}=d t$.

For $m \neq n$, denote by $\varphi_{\left(A_{n, i}-1, A_{m, i}+1\right)}(t)$ the rational $(L-1) M$-form defined for the matrix in $\mathcal{A}_{M}$ whose $(n, i)$ entry is $A_{n, i}-1$ and $(m, i)$ entry is $A_{m, i}+1$, and the other $(l, k)$ entries are $A_{l, k}$, and denote by $\varphi_{\left(A_{n, i}-1\right)}(t)$ the rational $(L-1) M$ form defined for the matrix in $\mathcal{A}_{M}$ whose $(n, i)$ entry is $A_{n, i}-1$, and the other $(l, k)$ entries are $A_{l, k}$.

By the definition of the covariant differential operator $\nabla$, we have

$$
X_{n}^{(i)}=\kappa \sum_{m=n}^{L-1} \operatorname{Sym}\left[\frac{\partial}{\partial t_{m}^{\left(S_{n}^{(i)}\right)}}\left(t_{m}^{\left(S_{n}^{(i)}\right)} \bar{\varphi}_{A}(t)\right)+\frac{\partial}{\partial t_{m}^{\left(S_{n}^{(i)}\right)}}(\log U(t)) t_{m}^{\left(S_{n}^{(i)}\right)} \bar{\varphi}_{A}(t)\right] d t,
$$

because $\log U(t)$ is invariant under the action of $\mathfrak{S}_{M}^{L-1}$.

Using the relations (4.7) and (4.8), we obtain by straightforward calculations

$$
\begin{aligned}
X_{n}^{(i)}= & \operatorname{Sym}\left[\left(\beta_{i}+\kappa\right) z_{i} \frac{t_{L-1}^{\left(S_{n}^{(i)}\right)}}{1-z_{i} t_{L-1}^{\left(S_{n}^{(i)}\right)}} \bar{\varphi}_{A}(t)\right] d t \\
& +\left(\sum_{m=n}^{L-1} \alpha_{m}-(L-1-n)\right) \varphi_{A}(t) \\
& +\left(1+\delta_{n, 1}(\gamma-1)\right) \sum_{m=n+1}^{L-1} \frac{A_{m, i}+1}{A_{n, i}} \varphi_{\left(A_{m, i}+1, A_{n, i}-1\right)}(t) \\
& +\left(1+\delta_{n, 1}(\gamma-1)\right) \frac{1}{z_{i}-1}\left(\frac{A_{0}+1}{A_{n, i}} \varphi_{\left(A_{n, i}-1\right)}(t)+\varphi_{A}(t) .\right. \\
& \left.+\sum_{\substack{m=1 \\
m \neq n}}^{L-1} \frac{A_{m, i}+1}{A_{n, i}} \varphi_{\left(A_{m, i}+1, A_{n, i}-1\right)}(t)\right) \\
& +\sum_{\substack{j=1 \\
j \neq i}}^{N} \frac{\beta_{j} z_{j}}{z_{i}-z_{j}}\left(\varphi_{A}(t)-\frac{A_{n, j}+1}{A_{n, i}} \varphi_{\left(A_{n, i}-1, A_{n, j}+1\right)}(t)\right)+Y_{n}^{(i)},
\end{aligned}
$$


where

$$
Y_{n}^{(i)}=\operatorname{Sym}\left[\sum_{m=n}^{L-1} t_{m}^{\left(S_{n}^{(i)}\right)} W_{n, m}^{(i)} \bar{\varphi}_{A}(t)\right] d t
$$

with

$$
W_{n, m}^{(i)}=\sum_{\substack{a=1 \\ a \neq S_{n}^{(i)}}}^{M}\left(\frac{-1}{t_{m}^{\left(S_{n}^{(i)}\right)}-t_{m-1}^{(a)}}+\frac{2}{t_{m}^{\left(S_{n}^{(i)}\right)}-t_{m}^{(a)}}+\frac{-1}{t_{m}^{\left(S_{n}^{(i)}\right)}-t_{m+1}^{(a)}}+\delta_{m, 1} \frac{1}{t_{1}^{\left(S_{n}^{(i)}\right)}-1}\right)
$$

We compute $Y_{n}^{(i)}$ in Lemmas 4.4, 4.5, 4.6 and 4.7. Owing to those lemmas, we obtain

$$
\kappa z_{i} \nabla_{i} \varphi_{A}(t)-\sum_{n=1}^{L-1} A_{n, i} X_{n}^{(i)}
$$

$=\left\{-\sum_{n=1}^{L-1} A_{n, i}\left(\sum_{m=n}^{L-1} \alpha_{m}-L+n-\beta_{i}+\sum_{m=1}^{n} A_{m, i}\right)\right.$

$-M \beta_{i}+\frac{1}{z_{i}-1}\left(A_{0}\left(\sum_{n=1}^{L-1} A_{n, i}-\beta_{i}\right)-\sum_{j=1}^{N} \sum_{n=1}^{L-1} A_{n, i} A_{n, j}+A_{1, i}(M-\gamma)\right)$

$\left.+\sum_{\substack{j=1 \\ j \neq i}}^{N} \frac{z_{j}}{z_{i}-z_{j}} \sum_{n=1}^{L-1}\left(A_{n, i}\left(\sum_{m=1}^{L-1} A_{m, j}+A_{n, j}-\beta_{j}\right)-\beta_{i} A_{n, j}\right)\right\} \varphi_{A}(t)$

$-\frac{A_{0}+1}{z_{i}-1} \sum_{n=1}^{L-1}\left(\sum_{j=1}^{N} A_{n, j}+\delta_{n, 1}(\gamma-M)\right) \varphi_{\left(A_{n, i}-1\right)}(t)$

$+\frac{z_{i}}{z_{i}-1}\left(\sum_{m=1}^{L-1} A_{m, i}-\beta_{i}\right) \sum_{n=1}^{L-1}\left(A_{n, i}+1\right) \varphi_{\left(A_{n, i}+1\right)}(t)$

$-\frac{1}{z_{i}-1} \sum_{n=1}^{L-1}\left(\sum_{j=1}^{N} A_{n, j}+\delta_{n, 1}(\gamma-M)\right)$

$\times\left(\sum_{m=1}^{n-1}\left(A_{m, i}+1\right) \varphi_{\left(A_{m, i}+1, A_{n, i}-1\right)}(t)+z_{i} \sum_{m=n+1}^{L-1}\left(A_{m, i}+1\right) \varphi_{\left(A_{m, i}+1, A_{n, i}-1\right)}(t)\right)$

$+\sum_{\substack{j=1 \\ j \neq i}}^{N} \frac{A_{n, j}+1}{z_{i}-z_{j}}\left(z_{j} \sum_{m=1}^{n-1}\left(A_{m, i}+1\right) \varphi_{\left(A_{m, j}-1, A_{m, i}+1, A_{n, i}-1, A_{n, j}+1\right)}(t)\right.$

$$
\left.+z_{i} \sum_{m=n+1}^{L-1}\left(A_{m, i}+1\right) \varphi_{\left(A_{m, j}-1, A_{m, i}+1, A_{n, i}-1, A_{n, j}+1\right)}(t)\right)
$$




$$
\begin{aligned}
& +\sum_{\substack{j=1 \\
j \neq i}}^{N} \frac{z_{i}}{z_{i}-z_{j}}\left(\beta_{i}-\sum_{m=1}^{L-1} A_{m, i}\right) \sum_{n=1}^{L-1}\left(A_{n, i}+1\right) \varphi_{\left(A_{n, j}-1, A_{n, i}+1\right)}(t) \\
& +\sum_{\substack{j=1 \\
j \neq i}}^{N} \frac{z_{j}}{z_{i}-z_{j}}\left(\beta_{j}-\sum_{m=1}^{L-1} A_{m, j}\right) \sum_{n=1}^{L-1}\left(A_{n, j}+1\right) \varphi_{\left(A_{n, i}-1, A_{n, j}+1\right)}(t),
\end{aligned}
$$

where the rational $(L-1) M$-form $\varphi_{\left(A_{m, j}-1, A_{m, i}+1, A_{n, i}-1, A_{n, j}+1\right)}(t)$ is defined for the matrix in $\mathcal{A}_{M}$ whose $(m, j),(m, i),(n, i),(n, j)$ entries are $A_{m, j}-1, A_{m, i}+1$, $A_{n, i}-1$, and $A_{n, j}+1$, respectively, and the other $(l, k)$ entries are $A_{l, k}$. Hence, for $A \in \mathcal{A}_{M}$, as an element in the twisted de Rham cohomology group $H^{(L-1) M}(T, \nabla)$, $\kappa \nabla_{i} \varphi_{A}(t)$ is expressed in terms of elements of $\left\{\varphi_{B}(t) \mid B \in \mathcal{A}_{M}\right\}$.

On the other hand, from Proposition 3.2, it is easy to see that the coefficient in $q^{A}$ of $H_{i} \Psi_{M}(\mathbf{q}, \mathbf{z})$ is equal to the hypergeometric pairing between the cycle $\Delta \in$ $H_{(L-1) M}\left(T, \mathcal{S}^{*}\right)$ and the right hand side of (4.11). This completes the proof.

\section{$\S 4.3$. Lemmas}

In the lemmas below, fix $1 \leq n \leq L-1,1 \leq i \leq N$ and $A \in \mathcal{A}_{M}$. For a triple $(n, i, A)$, the coboundary $X_{n}^{(i)}$ is defined by (4.9) and expressed as a linear combination of elements in $\left\{\varphi_{B}(t) \mid B \in \mathcal{A}_{M}\right\}$, and $Y_{n}^{(i)}$. In this subsection, we compute $Y_{n}^{(i)}$ and thus show that they can also be expressed as linear combinations of elements in $\left\{\varphi_{B}(t) \mid B \in \mathcal{A}_{M}\right\}$.

We decompose $Y_{n}^{(i)}$ as

$$
Y_{n}^{(i)}=\sum_{\substack{1 \leq j \leq N \\ 1 \leq \ell \leq L-1}}\left(Y_{n}^{(i)}\right)_{\ell, j}+\left(Y_{n}^{(i)}\right)_{0}
$$

and we compute $\left(Y_{n}^{(i)}\right)_{\ell, j}$ and $\left(Y_{n}^{(i)}\right)_{0}$, where for $\ell \neq n$ or $j \neq i$,

$$
\begin{aligned}
\left(Y_{n}^{(i)}\right)_{\ell, j} & =\operatorname{Sym}\left[A_{\ell, j} C\left(n, S_{n}^{(i)}, S_{\ell}^{(j)}\right) \bar{\varphi}_{A}(t)\right] d t, \\
\left(Y_{n}^{(i)}\right)_{n, i} & =\operatorname{Sym}\left[\left(A_{n, i}-1\right) C\left(n, S_{n}^{(i)}, S_{n}^{(i)}-1\right) \bar{\varphi}_{A}(t)\right] d t, \\
\left(Y_{n}^{(i)}\right)_{0} & =\operatorname{Sym}\left[A_{0} C\left(n, S_{n}^{(i)}, M-A_{0}+1\right) \bar{\varphi}_{A}(t)\right] d t,
\end{aligned}
$$

with, for $1 \leq a \neq b \leq M$,

$$
C(n, a, b)=\sum_{m=n}^{L-1} t_{m}^{(a)}\left(\frac{-1}{t_{m}^{(a)}-t_{m-1}^{(b)}}+\frac{2}{t_{m}^{(a)}-t_{m}^{(b)}}+\frac{-1}{t_{m}^{(a)}-t_{m+1}^{(b)}}\right)+\delta_{n, 1} \frac{t_{1}^{(a)}}{t_{1}^{(a)}-1} .
$$

Let the rational functions $f_{\ell, m}^{(j)}\left(t^{(a)}\right)$ be defined by

$$
f_{\ell, m}^{(j)}\left(t^{(a)}\right)=\frac{1}{1-z_{j} t_{L-1}^{(a)}} \sum_{\substack{k=1 \\ k \neq \ell, m}}^{L-1} \frac{1}{t_{k-1}^{(a)}-t_{k}^{(a)}} .
$$


Lemma 4.4. When $1 \leq \ell<n$, for $1 \leq j \neq i \leq N$, we have

$$
\begin{aligned}
\left(Y_{n}^{(i)}\right)_{\ell, j}= & \left(A_{\ell, i}+1\right) \varphi_{\left(A_{\ell, j}-1, A_{\ell, i}+1\right)}(t)+\frac{z_{j}}{z_{i}-z_{j}}\left(\left(A_{\ell, i}+1\right) \varphi_{\left(A_{\ell, j}-1, A_{\ell, i}+1\right)}(t)\right. \\
& -A_{\ell, j} \varphi_{A}(t)-\frac{\left(A_{\ell, i}+1\right)\left(A_{n, j}+1\right)}{A_{n, i}} \varphi_{\left(A_{\ell, j}-1, A_{\ell, i}+1, A_{n, i}-1, A_{n, j}+1\right)}(t) \\
& \left.+\frac{\left(A_{n, j}+1\right) A_{\ell, j}}{A_{n, i}} \varphi_{\left(A_{n, j}+1, A_{n, i}-1\right)}(t)\right),
\end{aligned}
$$

and for $j=i$, we have

$$
\left(Y_{n}^{(i)}\right)_{\ell, i}=A_{\ell, i} \varphi_{A}(t)
$$

Proof. It suffices to show that

$$
\begin{aligned}
& \operatorname{Sym}\left[C(n, 1,2) \frac{1}{t_{L-1}^{(1)}} f_{n}^{(i)}\left(t^{(1)}\right) \frac{1}{t_{L-1}^{(2)}} f_{\ell}^{(j)}\left(t^{(2)}\right)\right] \\
= & \operatorname{Sym}\left[\frac{1}{t_{L-1}^{(1)}} f_{n}^{(i)}\left(t^{(1)}\right) \frac{1}{t_{L-1}^{(2)}} f_{\ell}^{(i)}\left(t^{(2)}\right)\right] \\
& +\frac{z_{j}}{z_{i}-z_{j}} \operatorname{Sym}\left[\frac{1}{t_{L-1}^{(1)}} \frac{1}{t_{L-1}^{(2)}}\left(f_{n}^{(i)}\left(t^{(1)}\right)-f_{n}^{(j)}\left(t^{(1)}\right)\right)\left(f_{\ell}^{(i)}\left(t^{(2)}\right)-f_{\ell}^{(j)}\left(t^{(2)}\right)\right)\right]
\end{aligned}
$$

where $\operatorname{Sym}[f(t)]$ stands for $\sum_{\sigma \in \mathfrak{S}_{2}^{L-1}} \sigma(f(t))$ (see (4.3)), the rational functions $f_{n}^{(i)}\left(t^{(a)}\right)$ are defined in Definition 4.2 , and if $j=i$, then we understand that the second line of the right hand side of (4.12) disappears.

Firstly, we claim that for $n \leq k \leq L-2$,

$$
\begin{array}{r}
\operatorname{Sym}\left[\sum_{m=n}^{k}\left(\frac{-1}{t_{m}^{(1)}-t_{m-1}^{(2)}}+\frac{2}{t_{m}^{(1)}-t_{m}^{(2)}}+\frac{-1}{t_{m}^{(1)}-t_{m+1}^{(2)}}\right) \frac{t_{m}^{(1)}}{t_{L-1}^{(1)}} f_{n}^{(i)}\left(t^{(1)}\right) \frac{1}{t_{L-1}^{(2)}} f_{\ell}^{(j)}\left(t^{(2)}\right)\right] \\
=\operatorname{Sym}\left[\frac{1}{t_{k}^{(1)}-t_{k+1}^{(2)}} \frac{1}{t_{k+1}^{(1)}-t_{k}^{(2)}} \frac{t_{k+1}^{(1)}}{t_{L-1}^{(1)}} f_{n}^{(i)}\left(t^{(1)}\right) \frac{1}{t_{L-1}^{(2)}} f_{\ell, k+1}^{(j)}\left(t^{(2)}\right)\right] .
\end{array}
$$

We show (4.13) by induction. Let $k=n$. Then

$$
\begin{aligned}
& \operatorname{Sym}\left[\left(\frac{-1}{t_{n}^{(1)}-t_{n-1}^{(2)}}+\frac{1}{t_{n}^{(1)}-t_{n}^{(2)}}\right) \frac{t_{n}^{(1)}}{t_{L-1}^{(1)}} f_{n}^{(i)}\left(t^{(1)}\right) \frac{1}{t_{L-1}^{(2)}} f_{\ell}^{(j)}\left(t^{(2)}\right)\right] \\
= & \operatorname{Sym}\left[\frac{1}{t_{n-1}^{(2)}-t_{n}^{(1)}} \frac{1}{t_{n}^{(1)}-t_{n}^{(2)}} \frac{t_{n}^{(1)}}{t_{L-1}^{(1)}} f_{n}^{(i)}\left(t^{(1)}\right) \frac{1}{t_{L-1}^{(2)}} f_{\ell, n}^{(j)}\left(t^{(2)}\right)\right] \\
= & \operatorname{Sym}\left[\frac{-1}{t_{n}^{(1)}-t_{n}^{(2)}} \frac{1}{t_{n}^{(2)}-t_{n+1}^{(1)}} \frac{1}{t_{n}^{(1)}-t_{n+1}^{(2)}} \frac{t_{n}^{(2)}}{t_{L-1}^{(1)}} f_{n, n+1}^{(i)}\left(t^{(1)}\right) \frac{1}{t_{L-1}^{(2)}} f_{\ell, n+1}^{(j)}\left(t^{(2)}\right)\right],
\end{aligned}
$$

where in the last line, we interchanged $t_{n}^{(1)}$ with $t_{n}^{(2)}$ and 


$$
\begin{aligned}
& \operatorname{Sym}\left[\left(\frac{1}{t_{n}^{(1)}-t_{n}^{(2)}}+\frac{-1}{t_{n}^{(1)}-t_{n+1}^{(2)}}\right) \frac{t_{n}^{(1)}}{t_{L-1}^{(1)}} f_{n}^{(i)}\left(t^{(1)}\right) \frac{1}{t_{L-1}^{(2)}} f_{\ell}^{(j)}\left(t^{(2)}\right)\right] \\
& =\operatorname{Sym}\left[\frac{1}{t_{n}^{(1)}-t_{n}^{(2)}} \frac{1}{t_{n}^{(1)}-t_{n+1}^{(2)}} \frac{t_{n}^{(1)}}{t_{L-1}^{(1)}} f_{n}^{(i)}\left(t^{(1)}\right) \frac{1}{t_{L-1}^{(2)}} f_{\ell, n+1}^{(j)}\left(t^{(2)}\right)\right]
\end{aligned}
$$

Thus, the left hand side of (4.13) for $k=n$, that is, (4.14) plus (4.15), becomes the right hand side of (4.13) for $k=n$.

Suppose (4.13) holds for $k-1$. Then

$$
\begin{aligned}
\operatorname{Sym}[ & \left(\sum_{m=n}^{k-1} t_{m}^{(1)}\left(\frac{-1}{t_{m}^{(1)}-t_{m-1}^{(2)}}+\frac{2}{t_{m}^{(1)}-t_{m}^{(2)}}+\frac{-1}{t_{m}^{(1)}-t_{m+1}^{(2)}}\right)\right. \\
& \left.\left.\quad+t_{k}^{(1)}\left(\frac{-1}{t_{k}^{(1)}-t_{k-1}^{(2)}}+\frac{1}{t_{k}^{(1)}-t_{k}^{(2)}}\right)\right) \frac{1}{t_{L-1}^{(1)}} f_{n}^{(i)}\left(t^{(1)}\right) \frac{1}{t_{L-1}^{(2)}} f_{\ell}^{(j)}\left(t^{(2)}\right)\right] \\
= & \operatorname{Sym}\left[\frac{1}{t_{k}^{(1)}-t_{k}^{(2)}} \frac{1}{t_{k-1}^{(2)}-t_{k}^{(1)}} \frac{1}{t_{k-1}^{(1)}-t_{k}^{(2)}} \frac{t_{k}^{(1)}}{t_{L-1}^{(1)}} f_{n, k}^{(i)}\left(t^{(1)}\right) \frac{1}{t_{L-1}^{(2)}} f_{\ell, k}^{(j)}\left(t^{(2)}\right)\right] \\
= & \operatorname{Sym}\left[\frac{-1}{t_{k}^{(1)}-t_{k}^{(2)}} \frac{1}{t_{k}^{(2)}-t_{k+1}^{(1)}} \frac{1}{t_{k}^{(1)}-t_{k+1}^{(2)}} \frac{t_{k}^{(2)}}{t_{L-1}^{(1)}} f_{n, k+1}^{(i)}\left(t^{(1)}\right) \frac{1}{t_{L-1}^{(2)}} f_{\ell, k+1}^{(j)}\left(t^{(2)}\right)\right],
\end{aligned}
$$

where in the last line, we interchanged $t_{k}^{(1)}$ with $t_{k}^{(2)}$. Since

$$
\begin{aligned}
\operatorname{Sym}\left[\left(\frac{1}{t_{k}^{(1)}-t_{k}^{(2)}}+\frac{-1}{t_{k}^{(1)}-t_{k+1}^{(2)}}\right) \frac{t_{k}^{(1)}}{t_{L-1}^{(1)}} f_{n}^{(i)}\left(t^{(1)}\right) \frac{1}{t_{L-1}^{(2)}} f_{\ell}^{(j)}\left(t^{(2)}\right)\right] \\
=\operatorname{Sym}\left[\frac{1}{t_{k}^{(1)}-t_{k}^{(2)}} \frac{1}{t_{k}^{(1)}-t_{k+1}^{(2)}} \frac{t_{k}^{(1)}}{t_{L-1}^{(1)}} f_{n}^{(i)}\left(t^{(1)}\right) \frac{1}{t_{L-1}^{(2)}} f_{\ell, k+1}^{(j)}\left(t^{(2)}\right)\right],
\end{aligned}
$$

the left hand side of (4.13) for $k$ becomes the right hand side of (4.13) for $k$.

Secondly, using (4.13) for $k=L-2$, we have

$$
\begin{aligned}
& \operatorname{Sym}\left[\left(\sum_{m=n}^{L-2} t_{m}^{(1)}\left(\frac{-1}{t_{m}^{(1)}-t_{m-1}^{(2)}}+\frac{2}{t_{m}^{(1)}-t_{m}^{(2)}}+\frac{-1}{t_{m}^{(1)}-t_{m+1}^{(2)}}\right)\right.\right. \\
& \left.\left.\quad+t_{L-1}^{(1)}\left(\frac{-1}{t_{L-1}^{(1)}-t_{L-2}^{(2)}}+\frac{1}{t_{L-1}^{(1)}-t_{L-1}^{(2)}}\right)\right) \frac{1}{t_{L-1}^{(1)}} f_{n}^{(i)}\left(t^{(1)}\right) \frac{1}{t_{L-1}^{(2)}} f_{\ell}^{(j)}\left(t^{(2)}\right)\right] \\
= & \operatorname{Sym}\left[\frac{1}{t_{L-1}^{(1)}-t_{L-1}^{(2)}} \frac{1}{t_{L-2}^{(2)}-t_{L-1}^{(1)}} \frac{1}{t_{L-2}^{(1)}-t_{L-1}^{(2)}} f_{n, L-1}^{(i)}\left(t^{(1)}\right) \frac{1}{t_{L-1}^{(2)}} f_{\ell, L-1}^{(j)}\left(t^{(2)}\right)\right] \\
= & \operatorname{Sym}\left[\frac{-1}{t_{L-1}^{(1)}-t_{L-1}^{(2)}} f_{n}^{(j)}\left(t^{(1)}\right) \frac{1}{t_{L-1}^{(1)}} f_{\ell}^{(i)}\left(t^{(2)}\right)\right],
\end{aligned}
$$

where in the last line, we interchanged $t_{L-1}^{(1)}$ with $t_{L-1}^{(2)}$. Hence, the left hand side 
of $(4.12)$ is equal to

$$
\begin{gathered}
\operatorname{Sym}\left[\frac{-1}{t_{L-1}^{(1)}-t_{L-1}^{(2)}} f_{n}^{(j)}\left(t^{(1)}\right) \frac{1}{t_{L-1}^{(1)}} f_{\ell}^{(i)}\left(t^{(2)}\right)+\frac{1}{t_{L-1}^{(1)}-t_{L-1}^{(2)}} f_{n}^{(i)}\left(t^{(1)}\right) \frac{1}{t_{L-1}^{(2)}} f_{\ell}^{(j)}\left(t^{(2)}\right)\right] \\
\quad=\operatorname{Sym}\left[\frac{1}{t_{L-1}^{(1)}} \frac{1}{t_{L-1}^{(2)}} f_{n}^{(i)}\left(t^{(1)}\right) f_{\ell}^{(i)}\left(t^{(2)}\right) \frac{1-z_{j}\left(t_{L-1}^{(1)}+t_{L-1}^{(2)}\right)+z_{i} z_{j} t_{L-1}^{(1)} t_{L-1}^{(2)}}{\left(1-z_{j} t_{L-1}^{(1)}\right)\left(1-z_{j} t_{L-1}^{(2)}\right)}\right] .
\end{gathered}
$$

Therefore, the relation (4.12) holds.

Lemma 4.5. When $n<\ell \leq L-1$, for $1 \leq j \neq i \leq N$, we have

$$
\begin{aligned}
\left(Y_{n}^{(i)}\right)_{\ell, j} & =-A_{\ell, j}\left(\frac{\delta_{n, 1}}{z_{i}-1}+\frac{z_{j}}{z_{i}-z_{j}}\right) \varphi_{A}(t)+\left(A_{\ell, i}+1\right) \frac{z_{i}}{z_{i}-z_{j}} \varphi_{\left(A_{\ell, j}-1, A_{\ell, i}+1\right)}(t) \\
& -\frac{\left(A_{\ell, i}+1\right)\left(A_{n, j}+1\right)}{A_{n, i}} \frac{z_{i}}{z_{i}-z_{j}} \varphi_{\left(A_{\ell, j}-1, A_{\ell, i}+1, A_{n, i}-1, A_{n, j}+1\right)}(t) \\
& +\frac{\left(A_{n, j}+1\right) A_{\ell, j}}{A_{n, i}} \frac{z_{j}}{z_{i}-z_{j}} \varphi_{\left(A_{n, j}+1, A_{n, i}-1\right)}(t) \\
& -\frac{A_{\ell, j}}{A_{n, i}} \frac{\delta_{n, 1}}{z_{i}-1}\left(\left(A_{0}+1\right) \varphi_{\left(A_{n, i}-1\right)}(t)+z_{i} \sum_{m=2}^{L-1}\left(A_{m, i}+1\right) \varphi_{\left(A_{m, i}+1, A_{1, i}-1\right)}(t)\right)
\end{aligned}
$$

and for $j=i$, we have

$$
\begin{aligned}
\left(Y_{n}^{(i)}\right)_{\ell, i}=-\frac{\delta_{n, 1} A_{\ell, i}}{z_{i}-1}\left(\varphi_{A}(t)+\right. & \frac{\left(A_{0}+1\right)}{A_{1, i}} \varphi_{\left(A_{1, i}-1\right)}(t) \\
& \left.+z_{i} \sum_{m=2}^{L-1} \frac{\left(A_{m, i}+1\right)}{A_{1, i}} \varphi_{\left(A_{m, i}+1, A_{1, i}-1\right)}(t)\right) .
\end{aligned}
$$

Proof. It suffices to show that for $n \geq 2$,

$$
\begin{aligned}
& \operatorname{Sym}\left[C(n, 1,2) \frac{1}{t_{L-1}^{(1)}} f_{n}^{(i)}\left(t^{(1)}\right) \frac{1}{t_{L-1}^{(2)}} f_{\ell}^{(j)}\left(t^{(2)}\right)\right] \\
= & \frac{1}{z_{i}-z_{j}} \operatorname{Sym}\left[\frac{1}{t_{L-1}^{(1)}} \frac{1}{t_{L-1}^{(2)}}\left(f_{n}^{(i)}\left(t^{(1)}\right)-f_{n}^{(j)}\left(t^{(1)}\right)\right)\left(z_{i} f_{\ell}^{(i)}\left(t^{(2)}\right)-z_{j} f_{\ell}^{(j)}\left(t^{(2)}\right)\right)\right],
\end{aligned}
$$

and for $n=1$,

$$
\text { (4.17) } \begin{aligned}
& \operatorname{Sym}\left[C(1,1,2) \frac{1}{t_{L-1}^{(1)}} f_{1}^{(i)}\left(t^{(1)}\right) \frac{1}{t_{L-1}^{(2)}} f_{\ell}^{(j)}\left(t^{(2)}\right)\right] \\
= & \frac{1}{z_{i}-z_{j}} \operatorname{Sym}\left[\frac{1}{t_{L-1}^{(1)}} \frac{1}{t_{L-1}^{(2)}}\left(f_{1}^{(i)}\left(t^{(1)}\right)-f_{1}^{(j)}\left(t^{(1)}\right)\right)\left(z_{i} f_{\ell}^{(i)}\left(t^{(2)}\right)-z_{j} f_{\ell}^{(j)}\left(t^{(2)}\right)\right)\right] \\
+ & \frac{1}{z_{i}-1} \operatorname{Sym}\left[\frac{1}{t_{L-1}^{(1)}}\left(f_{0}\left(t^{(1)}\right)-f_{1}^{(i)}\left(t^{(1)}\right)-z_{i} \sum_{m=2}^{L-1} f_{m}^{(i)}\left(t^{(1)}\right)\right) \frac{1}{t_{L-1}^{(2)}} f_{\ell}^{(j)}\left(t^{(2)}\right)\right]
\end{aligned}
$$


where $\operatorname{Sym}[f(t)]$ stands for $\sum_{\sigma \in \mathfrak{S}_{2}^{L-1}} \sigma(f(t))$ (see (4.3)), and if $j=i$, then we understand that the right hand side of (4.16) and the first line of the right hand side of (4.17) are zero.

We shall show (4.16). Firstly, using (4.13) for $k=\ell-2$, we have

$$
\begin{aligned}
& \operatorname{Sym}[\left(\sum_{m=n}^{\ell-2} t_{m}^{(1)}\left(\frac{-1}{t_{m}^{(1)}-t_{m-1}^{(2)}}+\frac{2}{t_{m}^{(1)}-t_{m}^{(2)}}+\frac{-1}{t_{m}^{(1)}-t_{m+1}^{(2)}}\right)\right. \\
&\left.\left.+t_{\ell-1}^{(1)}\left(\frac{-1}{t_{\ell-1}^{(1)}-t_{\ell-2}^{(1)}}+\frac{1}{t_{\ell-1}^{(1)}-t_{\ell-1}^{(2)}}\right)\right) \frac{1}{t_{L-1}^{(1)}} f_{n}^{(i)}\left(t^{(1)}\right) \frac{1}{t_{L-1}^{(2)}} f_{\ell}^{(j)}\left(t^{(2)}\right)\right] \\
&=\operatorname{Sym}\left[\frac{1}{t_{\ell-1}^{(1)}-t_{\ell-1}^{(2)}} \frac{1}{t_{\ell-2}^{(1)}-t_{\ell-1}^{(2)}} \frac{1}{t_{\ell-2}^{(2)}-t_{\ell-1}^{(1)}} \frac{t_{\ell-1}^{(1)}}{t_{L-1}^{(1)}} f_{n, \ell-1}^{(i)}\left(t^{(1)}\right) \frac{1}{t_{L-1}^{(2)}} f_{\ell-1, \ell}^{(j)}\left(t^{(2)}\right)\right] \\
&=\operatorname{Sym}\left[\frac{-1}{t_{\ell-1}^{(1)}-t_{\ell-1}^{(2)}} \frac{1}{t_{\ell-1}^{(2)}-t_{\ell}^{(1)}} \frac{t_{\ell-1}^{(2)}}{t_{L-1}^{(1)}} f_{n, \ell}^{(i)}\left(t^{(1)}\right) \frac{1}{t_{L-1}^{(2)}} f_{\ell}^{(j)}\left(t^{(2)}\right)\right],
\end{aligned}
$$

where in the last line, we interchanged $t_{\ell-1}^{(1)}$ with $t_{\ell-1}^{(2)}$. Thus, (4.18) is equal to

$$
-\operatorname{Sym}\left[\left(\frac{t_{\ell-1}^{(1)}}{t_{\ell-1}^{(1)}-t_{\ell-1}^{(2)}}+\frac{-t_{\ell}^{(1)}}{t_{\ell}^{(1)}-t_{\ell-1}^{(2)}}\right) \frac{1}{t_{L-1}^{(1)}} f_{n}^{(i)}\left(t^{(1)}\right) \frac{1}{t_{L-1}^{(2)}} f_{\ell}^{(j)}\left(t^{(2)}\right)\right] .
$$

Secondly, we claim that for $\ell \leq k \leq L-2$, we have

$$
\begin{array}{r}
\operatorname{Sym}\left[\sum_{m=\ell}^{k}\left(\frac{-t_{m+1}^{(1)}}{t_{m+1}^{(1)}-t_{m}^{(2)}}+\frac{2 t_{m}^{(1)}}{t_{m}^{(1)}-t_{m}^{(2)}}+\frac{-t_{m-1}^{(1)}}{t_{m-1}^{(1)}-t_{m}^{(2)}}\right) \frac{1}{t_{L-1}^{(1)}} f_{n}^{(i)}\left(t^{(1)}\right) \frac{1}{t_{L-1}^{(2)}} f_{\ell}^{(j)}\left(t^{(2)}\right)\right] \\
=\operatorname{Sym}\left[\frac{1}{t_{k}^{(1)}-t_{k+1}^{(2)}} \frac{1}{t_{k}^{(2)}-t_{k+1}^{(1)}} \frac{1}{t_{L-1}^{(1)}} f_{n, k+1}^{(i)}\left(t^{(1)}\right) \frac{t_{k+1}^{(2)}}{t_{L-1}^{(2)}} f_{\ell}^{(j)}\left(t^{(2)}\right)\right] .
\end{array}
$$

We can prove (4.19) by induction; we omit the details.

Using (4.19) for $k=L-2$, we have

$$
\begin{aligned}
\operatorname{Sym}[ & \left(\sum_{m=\ell}^{L-2}\left(\frac{-t_{m+1}^{(1)}}{t_{m+1}^{(1)}-t_{m}^{(2)}}+\frac{2 t_{m}^{(1)}}{t_{m}^{(1)}-t_{m}^{(2)}}+\frac{-t_{m-1}^{(1)}}{t_{m-1}^{(1)}-t_{m}^{(2)}}\right)\right. \\
& \left.\left.\quad+\frac{t_{L-1}^{(1)}}{t_{L-1}^{(1)}-t_{L-1}^{(2)}}+\frac{-t_{L-2}^{(1)}}{t_{L-2}^{(1)}-t_{L-1}^{(2)}}\right) \frac{1}{t_{L-1}^{(1)}} f_{n}^{(i)}\left(t^{(1)}\right) \frac{1}{t_{L-1}^{(2)}} f_{\ell}^{(j)}\left(t^{(2)}\right)\right] \\
& =\operatorname{Sym}\left[\frac{1}{t_{L-1}^{(1)}-t_{L-1}^{(2)}} \frac{1}{t_{L-2}^{(1)}-t_{L-1}^{(2)}} \frac{1}{t_{L-2}^{(2)}-t_{L-1}^{(1)}} \frac{1}{t_{L-1}^{(1)}} f_{n, L-1}^{(i)}\left(t^{(1)}\right) f_{\ell, L-1}^{(j)}\left(t^{(2)}\right)\right] \\
= & \operatorname{Sym}\left[\frac{-1}{t_{L-1}^{(1)}-t_{L-1}^{(2)}} \frac{1}{t_{L-1}^{(2)}} f_{n}^{(j)}\left(t^{(1)}\right) f_{\ell}^{(i)}\left(t^{(2)}\right)\right],
\end{aligned}
$$


where in the last line, we interchanged $t_{L-1}^{(1)}$ with $t_{L-1}^{(2)}$. Hence, the left hand side of (4.16) is equal to

$$
\begin{array}{r}
\operatorname{Sym}\left[\frac{-1}{t_{L-1}^{(1)}-t_{L-1}^{(2)}} f_{n}^{(j)}\left(t^{(1)}\right) \frac{1}{t_{L-1}^{(2)}} f_{\ell}^{(i)}\left(t^{(2)}\right)+\frac{1}{t_{L-1}^{(1)}-t_{L-1}^{(2)}} f_{n}^{(i)}\left(t^{(1)}\right) \frac{1}{t_{L-1}^{(2)}} f_{\ell}^{(j)}\left(t^{(2)}\right)\right] \\
=\left(z_{i}-z_{j}\right) \operatorname{Sym}\left[\frac{1}{1-z_{j} t_{L-1}^{(1)}} f_{n}^{(i)}\left(t^{(1)}\right) \frac{1}{t_{L-1}^{(2)}\left(1-z_{i} t_{L-1}^{(2)}\right)} f_{l}^{(j)}\right] .
\end{array}
$$

Therefore, the relation (4.16) holds.

We shall show (4.17). We compute

$$
\begin{aligned}
\text { L.H.S. of }(4.17)= & \operatorname{Sym}\left[\left(\frac{-t_{1}^{(1)}}{t_{1}^{(1)}-1}+C(1,1,2)\right) \frac{1}{t_{L-1}^{(1)}} f_{1}^{(i)}\left(t^{(1)}\right) \frac{1}{t_{L-1}^{(2)}} f_{\ell}^{(j)}\left(t^{(2)}\right)\right] \\
& +\operatorname{Sym}\left[\frac{t_{1}^{(1)}}{t_{1}^{(1)}-1} \frac{1}{t_{L-1}^{(1)}} f_{1}^{(i)}\left(t^{(1)}\right) \frac{1}{t_{L-1}^{(2)}} f_{\ell}\left(t^{(2)}\right)\right] .
\end{aligned}
$$

The first line of the right hand side becomes the first line of the right hand side of (4.17) in the same way as in the proof of (4.16). On the other hand,

$$
\begin{aligned}
& \operatorname{Sym}\left[\frac{1}{t_{1}^{(1)}-1} \frac{t_{1}^{(1)}}{t_{L-1}^{(1)}} f_{1}^{(i)}\left(t^{(1)}\right) \frac{1}{t_{L-1}^{(2)}} f_{\ell}\left(t^{(2)}\right)\right] \\
& =\operatorname{Sym}\left[\frac{t_{L-1}^{(1)}+\sum_{m=2}^{L-1}\left(t_{m-1}^{(1)}-t_{m}^{(1)}\right)}{t_{1}^{(1)}-1} \frac{1}{t_{L-1}^{(1)}} f_{1}^{(i)}\left(t^{(1)}\right) \frac{1}{t_{L-1}^{(2)}} f_{\ell}\left(t^{(2)}\right)\right] \\
& =\operatorname{Sym}\left[\frac{1}{z_{i}-1} \frac{1}{t_{L-1}^{(1)}}\left(f_{0}\left(t^{(1)}\right)-f_{1}\left(t^{(1)}\right)-z_{i} \sum_{m=2}^{L-1} f_{m}^{(i)}\left(t^{(1)}\right)\right) \frac{1}{t_{L-1}^{(2)}} f_{\ell}\left(t^{(2)}\right)\right] .
\end{aligned}
$$

Therefore, (4.17) holds.

Lemma 4.6. For $1 \leq j \neq i \leq L-1$, we have

$$
\begin{aligned}
\left(Y_{n}^{(i)}\right)_{n, j}= & A_{n, j}\left(\frac{1-\delta_{n, 1}}{z_{i}-1}-\frac{2 z_{j}}{z_{i}-z_{j}}\right) \varphi_{A}(t)+\frac{1-\delta_{n, 1}}{z_{i}-1} \frac{\left(A_{0}+1\right) A_{n, j}}{A_{n, i}} \varphi_{\left(A_{n, i}-1\right)}(t) \\
& +\frac{1-\delta_{n, 1}}{z_{i}-1} \frac{A_{n, j}}{A_{n, i}}\left(\sum_{m=1}^{n-1}\left(A_{m, i}+1\right) \varphi_{\left(A_{m, i}+1, A_{n, i}-1\right)}(t)\right. \\
& \left.+z_{i} \sum_{m=n+1}^{L-1}\left(A_{m, i}+1\right) \varphi_{\left(A_{m, i}+1, A_{n, i}-1\right)}(t)\right) \\
& +\left(A_{n, i}+1\right) \frac{z_{i}}{z_{i}-z_{j}} \varphi_{\left(A_{n, j}-1, A_{n, i}+1\right)}(t) \\
& +\frac{\left(A_{n, j}+1\right) A_{n, j}}{A_{n, i}} \frac{z_{j}}{z_{i}-z_{j}} \varphi_{\left(A_{n, j}+1, A_{n, i}-1\right)}(t),
\end{aligned}
$$


and for $j=i$, we have

$$
\begin{aligned}
\left(Y_{n}^{(i)}\right)_{n, i}=\left(A_{n, i}-1\right)\left(\frac{z_{i}-\delta_{n, 1}}{z_{i}-1}\right) \varphi_{A}(t) & +\frac{1-\delta_{n, 1}}{z_{i}-1} \frac{\left(A_{0}+1\right)\left(A_{n, i}-1\right)}{A_{n, i}} \varphi_{\left(A_{n, i}-1\right)}(t) \\
+ & \frac{1-\delta_{n, 1}}{z_{i}-1} \frac{A_{n, i}-1}{A_{n, i}}\left(\sum_{m=1}^{n-1}\left(A_{m, i}+1\right) \varphi_{\left(A_{m, i}+1, A_{n, i}-1\right)}(t)\right. \\
& \left.+z_{i} \sum_{m=n+1}^{L-1}\left(A_{m, i}+1\right) \varphi_{\left(A_{m, i}+1, A_{n, i}-1\right)}(t)\right) .
\end{aligned}
$$

Proof. It suffices to show that

$$
\begin{aligned}
& \text { 20) } \operatorname{Sym}\left[C(n, 1,2) \frac{1}{t_{L-1}^{(1)}} f_{n}^{(i)}\left(t^{(1)}\right) \frac{1}{t_{L-1}^{(2)}} f_{n}^{(j)}\left(t^{(2)}\right)\right] \\
& =\operatorname{Sym}\left[\frac{1}{t_{L-1}^{(1)}} f_{n}^{(i)}\left(t^{(1)}\right) \frac{1}{t_{L-1}^{(2)}} f_{n}^{(i)}\left(t^{(2)}\right)\right] \\
& +\frac{1-\delta_{n, 1}}{z_{i}-1} \operatorname{Sym}\left[\left(-f_{0}\left(t^{(1)}\right)+\sum_{m=1}^{n} f_{m}^{(i)}\left(t^{(1)}\right)+z_{i} \sum_{m=n+1}^{L-1} f_{m}^{(i)}\left(t^{(1)}\right)\right) \frac{f_{n}^{(j)}\left(t^{(2)}\right)}{t_{L-1}^{(1)} t_{L-1}^{(2)}}\right] \\
& +\frac{z_{j}}{z_{i}-z_{j}} \operatorname{Sym}\left[\frac{1}{t_{L-1}^{(1)}} \frac{1}{t_{L-1}^{(2)}}\left(f_{n}^{(i)}\left(t^{(1)}\right)-f_{n}^{(j)}\left(t^{(1)}\right)\right)\left(f_{n}^{(i)}\left(t^{(2)}\right)-f_{n}^{(j)}\left(t^{(2)}\right)\right)\right]
\end{aligned}
$$

where $\operatorname{Sym}[f(t)]$ stands for $\sum_{\sigma \in \mathfrak{S}_{2}^{L-1}} \sigma(f(t))$ (see (4.3)), and if $j=i$, then we understand that the third line of the right hand side of (4.20) disappears.

Firstly, we have

$$
\begin{aligned}
\operatorname{Sym}\left[\frac{-t_{n}^{(1)}}{t_{n}^{(1)}-t_{n-1}^{(2)}} \frac{1}{t_{L-1}^{(1)}} f_{n}^{(i)}\left(t^{(1)}\right) \frac{1}{t_{L-1}^{(2)}} f_{n}^{(j)}\left(t^{(2)}\right)\right] \\
\quad=\operatorname{Sym}\left[\frac{t_{L-1}^{(1)}+\sum_{m=n+1}^{L-1}\left(t_{m-1}^{(1)}-t_{m}^{(1)}\right)}{t_{n}^{(1)}-t_{n-1}^{(1)}} \frac{1}{t_{L-1}^{(1)}} f_{n}^{(i)}\left(t^{(1)}\right) \frac{1}{t_{L-1}^{(2)}} f_{n}^{(j)}\left(t^{(2)}\right)\right] \\
=\frac{1}{z_{i}-1} \operatorname{Sym}\left[\left(-f_{0}\left(t^{(1)}\right)+\sum_{m=1}^{n} f_{m}^{(i)}\left(t^{(1)}\right)+z_{i} \sum_{m=n+1}^{L-1} f_{m}^{(i)}\left(t^{(1)}\right)\right) \frac{f_{n}^{(j)}\left(t^{(2)}\right)}{t_{L-1}^{(1)} t_{L-1}^{(2)}}\right] .
\end{aligned}
$$

Secondly, we notice that for $n \leq m \leq L-2$, we have

$$
\operatorname{Sym}\left[\left(\frac{-t_{m+1}^{(1)}}{t_{m+1}^{(1)}-t_{m}^{(2)}}+\frac{2 t_{m}^{(1)}}{t_{m}^{(1)}-t_{m}^{(2)}}+\frac{-t_{m-1}^{(1)}}{t_{m-1}^{(1)}-t_{m}^{(2)}}\right) \frac{1}{t_{L-1}^{(1)}} f_{n}^{(i)}\left(t^{(1)}\right) \frac{1}{t_{L-1}^{(2)}} f_{n}^{(j)}\left(t^{(2)}\right)\right]=0 .
$$


Thirdly, we compute the remaining term as follows:

$$
\begin{aligned}
& \operatorname{Sym}\left[\frac{2}{t_{L-1}^{(1)}-t_{L-1}^{(2)}} f_{n}^{(i)}\left(t^{(1)}\right) \frac{1}{t_{L-1}^{(2)}} f_{n}^{(j)}\left(t^{(2)}\right)\right] \\
& =\operatorname{Sym}\left[\frac{-1}{t_{L-1}^{(1)}-t_{L-1}^{(2)}} f_{n}^{(j)}\left(t^{(1)}\right) \frac{1}{t_{L-1}^{(1)}} f_{n}^{(i)}\left(t^{(2)}\right)+\frac{1}{t_{L-1}^{(1)}-t_{L-1}^{(2)}} f_{n}^{(i)}\left(t^{(1)}\right) \frac{1}{t_{L-1}^{(2)}} f_{n}^{(j)}\left(t^{(2)}\right)\right] \\
& =\operatorname{Sym}\left[\frac{1}{t_{L-1}^{(1)}} \frac{1}{t_{L-1}^{(2)}} f_{n}^{(i)}\left(t^{(1)}\right) f_{n}^{(i)}\left(t^{(2)}\right) \frac{1-z_{j}\left(t_{L-1}^{(1)}+t_{L-1}^{(2)}\right)+z_{i} z_{j} t_{L-1}^{(1)} t_{L-1}^{(2)}}{\left(1-z_{j} t_{L-1}^{(1)}\right)\left(1-z_{j} t_{L-1}^{(2)}\right)}\right] .
\end{aligned}
$$

Therefore, the relation (4.20) holds.

Lemma 4.7. We have

$$
\begin{aligned}
\left(Y_{n}^{(i)}\right)_{0}= & -\frac{1+\delta_{n, 1}}{z_{i}-1} A_{0} \varphi_{A}(t)+\frac{z_{i}}{z_{i}-1} \sum_{m=1}^{L-1}\left(A_{m, i}+1\right) \varphi_{\left(A_{m, i}+1\right)}(t) \\
& -\delta_{n, 1} \frac{1}{z_{i}-1} \frac{A_{0}\left(A_{0}+1\right)}{A_{1, i}} \varphi_{\left(A_{1, i}-1\right)}(t) \\
& -\delta_{n, 1} \frac{z_{i}}{z_{i}-1} \sum_{m=2}^{L-1} \frac{A_{0}\left(A_{m, i}+1\right)}{A_{1, i}} \varphi_{\left(A_{m, i}+1, A_{1, i}-1\right)}(t) .
\end{aligned}
$$

Proof. It suffices to show that

$$
\begin{aligned}
& \operatorname{Sym}\left[C(n, 1,2) \frac{1}{t_{L-1}^{(1)}} f_{n}^{(i)}\left(t^{(1)}\right) \frac{1}{t_{L-1}^{(2)}} f_{0}\left(t^{(2)}\right)\right] \\
= & \operatorname{Sym}\left[\frac{1}{\left(z_{i}-1\right) t_{L-1}^{(1)} t_{L-1}^{(2)}} f_{n}^{(i)}\left(t^{(1)}\right)\left(-\left(1+\delta_{n, 1}\right) f_{0}\left(t^{(2)}\right)+z_{i} \sum_{m=1}^{L-1} f_{m}^{(i)}\left(t^{(2)}\right)\right)\right] \\
& +\delta_{n, 1} \operatorname{Sym}\left[\frac{1}{\left(z_{i}-1\right) t_{L-1}^{(1)} t_{L-1}^{(2)}} f_{0}\left(t^{(2)}\right)\left(f_{0}\left(t^{(1)}\right)-z_{i} \sum_{m=2}^{L-1} f_{m}^{(i)}\left(t^{(1)}\right)\right)\right]
\end{aligned}
$$

where $\operatorname{Sym}[f(t)]$ stands for $\sum_{\sigma \in \mathfrak{S}_{2}^{L-1}} \sigma(f(t))$ and the rational functions $f_{0}\left(t^{(a)}\right)$ are defined in Definition 4.2.

Firstly, using (4.13) for $l=0$ and $k=L-2$, we have

$$
\begin{gathered}
\operatorname{Sym}\left[\left(C(n, 1,2)+\delta_{n, 1} \frac{-1}{t_{1}^{(1)}-1} \frac{t_{1}^{(1)}}{t_{L-1}^{(1)}}\right) \frac{1}{t_{L-1}^{(1)}} f_{n}^{(i)}\left(t^{(1)}\right) \frac{1}{t_{L-1}^{(2)}} f_{0}\left(t^{(2)}\right)\right] \\
=\operatorname{Sym}\left[\frac{-1}{t_{L-1}^{(1)}-t_{L-1}^{(2)}} \frac{1-z_{i} t_{L-1}^{(1)}}{1-z_{i} t_{L-1}^{(2)}} f_{n}^{(i)}\left(t^{(1)}\right) \frac{1}{t_{L-1}^{(1)}} f_{0}\left(t^{(2)}\right)\right. \\
\left.+\frac{1}{t_{L-1}^{(1)}-t_{L-1}^{(2)}} f_{n}^{(i)}\left(t^{(1)}\right) \frac{1}{t_{L-1}^{(2)}} f_{0}\left(t^{(2)}\right)\right]
\end{gathered}
$$




$$
=\operatorname{Sym}\left[\frac{1}{t_{L-1}^{(1)}} f_{n}^{(i)}\left(t^{(1)}\right) \frac{1}{t_{L-1}^{(2)}\left(1-z_{i} t_{L-1}^{(2)}\right)} f_{0}\left(t^{(2)}\right)\right] \text {. }
$$

Secondly, we have

$$
\begin{aligned}
\operatorname{Sym}\left[\frac{1}{t_{1}^{(1)}-1} \frac{t_{1}^{(1)}}{t_{L-1}^{(1)}} f_{1}^{(i)}\left(t^{(1)}\right) \frac{1}{t_{L-1}^{(2)}} f_{0}\left(t^{(2)}\right)\right] \\
\quad=\operatorname{Sym}\left[\frac{t_{L-1}^{(1)}+\sum_{m=2}^{L-1}\left(t_{m-1}^{(1)}-t_{m}^{(1)}\right)}{t_{1}^{(1)}-1} \frac{1}{t_{L-1}^{(1)}} f_{1}^{(i)}\left(t^{(1)}\right) \frac{1}{t_{L-1}^{(2)}} f_{0}\left(t^{(2)}\right)\right] \\
=\operatorname{Sym}\left[\frac{1}{z_{i}-1} \frac{1}{t_{L-1}^{(1)}}\left(f_{0}\left(t^{(1)}\right)-f_{1}\left(t^{(1)}\right)-z_{i} \sum_{m=2}^{L-1} f_{m}^{(i)}\left(t^{(1)}\right)\right) \frac{1}{t_{L-1}^{(2)}} f_{0}\left(t^{(2)}\right)\right] .
\end{aligned}
$$

Therefore, the relation (4.21) holds.

\section{Acknowledgements}

The author is grateful to T. Tsuda and Y. Yamada for helpful discussions. This work was partially supported by Grant-in-Aid for Japan Society for the Promotion of Science (No. 22-2255).

\section{References}

[1] H. Awata, A. Tsuchiya and Y. Yamada, Integral formulas for the WZNW correlation functions, Nuclear Phys. B365 (1991), 680-696. MR 1136712

[2] J. Björk, Rings of differential operators, North-Holland, 1979. Z Zbl 0499.13009 MR 0549189

[3] K. Fuji and T. Suzuki, Drinfeld-Sokolov hierarchies of type A and fourth order Painlevé systems, Funkcial. Ekvac. 53 (2010), 143-167. Zbl 1202.34156 MR 2668518

[4] R. Garnier, Sur des équations différentielles du troisième ordre dont l'intégrale générale est uniforme et sur une classe d'équations nouvelles d'ordre supérieur dont l'intégrale générale a ses points critiques fixes, Ann. Sci. Ecole Norm. Sup. 29 (1912), 1-126. JFM 43.0382.01 MR 1509146

[5] J. Harnad, Quantum isomonodromic deformations and the Knizhnik-Zamolodchikov equations, in Symmetries and integrability of difference equations (Estérel, PQ, 1994), CRM Proc. Lecture Notes 9, Amer. Math. Soc., 1996, 155-161. Zbl 0860.35123 MR 1416835

[6] H. Kimura and K. Okamoto, On the polynomial Hamiltonian structure of the Garnier system, J. Math. Pures Appl. 63 (1984), 129-146. Zbl 0562.34004 MR 0776915

[7] H. Nagoya, Quantum Painlevé systems of type $A_{l}^{(1)}$, Int. J. Math. 15 (2004), 1007-1031. Zbl 1066.34087 MR 2106261

[8] _ A quantization of the sixth Painlevé equation, in Noncommutativity and singularities, Adv. Stud. Pure Math. 55, Math. Soc. Japan, 2009, 291-298. Zbl 1185.34138 MR 2463505

[9] Hypergeometric solutions to Schrödinger equations for the quantum Painlevé equations, J. Math. Phys. 52 (2011), no. 8, 083509, 16 pp. MR 2858065 
[10] _ Realizations of affine Weyl group symmetries on the quantum Painlevé equations by fractional calculus, Lett. Math. Phys. 102 (2012), 297-321. Zbl 1260.81118 MR 2989486

[11] K. Okamoto and H. Kimura, On particular solutions of the Garnier systems and the hypergeometric functions of several variables, Quart. J. Math. 37 (1986), 61-80. Zbl 0597.35114 MR 0830631

[12] N. Reshetikhin, The Knizhnik-Zamolodchikov system as a deformation of the isomonodromy problem, Lett. Math. Phys. 26 (1992), 167-177. Zbl 0776.17016 MR 1199740

[13] V. V. Schechtman and A. Varchenko, Hypergeometric solutions of Knizhnik-Zamolodchikov equations, Lett. Math. Phys. 20 (1990), 279-283. Zbl 0719.35079 MR 1077959

[14] T. Suzuki, A class of higher order Painlevé systems arising from integrable hierarchies of type A, Contemp. Math., to appear.

[15] _ A particular solution of a Painlevé system in terms of the hypergeometric function ${ }_{n+1} F_{n}$, SIGMA 6 (2010), paper 078, 11 pp. Zbl 1220.34112 MR 2769937

[16] J. Teschner, Quantization of the Hitchin moduli spaces, Liouville theory, and the geometric Langlands correspondence I, Adv. Theor. Math. Phys. 15 (2011), 471-564. Zbl pre06118033 MR 2924236

[17] T. Tsuda, Hypergeometric solution of a certain polynomial Hamiltonian system of isomonodromy type, Quart. J. Math. 63 (2010), 489-505. Zbl pre06045455 MR 2925302

[18] _ UC hierarchy and monodromy preserving deformation, J. Reine Angew. Math., in press, doi:10.1515/crelle-2012-0022.

[19] Y. Yamada, A quantum isomonodromy equation and its application to $\mathcal{N}=2 S U(N)$ gauge theories, J. Phys. A 44 (2011), no. 5, 055403, 9 pp. Zbl 1208.81150 MR 2763466 